\title{
A variational calculation of vibrational levels of vinyl radical
}

\author{
Xiao-Gang Wang* and Tucker Carrington $\mathrm{Jr}^{\dagger}$ \\ Chemistry Department, Queen's University, \\ Kingston, Ontario KYL 3N6, Canada
}

(Dated: March 06, 2020)

\begin{abstract}
We report vibrational energy levels of vinyl radical (VR) that are computed with a Lanczos eigensolver and contracted basis. Many of the levels of the two previous VR variational calculations differ significantly and differ also from those reported in this paper. We identify the source of and correct symmetry errors on the potential energy surfaces used in the previous calculations. VR has two equivalent equilibrium structures. By plotting wavefunction cuts, we show that two tunnelling paths play an important role. Using computed wavefunctions it is possible to assign many states and thereby to determine tunnelling splittings that are compared with their experimental counterparts. Our computed red shift of the hot band at $2897.23 \mathrm{~cm}^{-1}$, observed by F. Dong, M. Roberts and D. J. Nesbitt [J Chem. Phys. 128, 044305 (2008)], is $4.47 \mathrm{~cm}^{-1}$ which is close to the experimental value of $4.63 \mathrm{~cm}^{-1}$.
\end{abstract}

\footnotetext{
*Electronic address: xgwang.dalian@gmail.com

${ }^{\dagger}$ Electronic address: Tucker.Carrington@queensu.ca, Fax :613-533-6669
} 


\section{INTRODUCTION}

In recent years, the vinyl radical (VR), $\mathrm{H}_{2} \mathrm{C}_{\beta}-\mathrm{C}_{\alpha} \mathrm{H}$, has been studied by both theoretical[17] and experimental groups. [8-16] VR has two equivalent $C_{s}$ equilibrium structures connected by an in-plane tunnelling path. The saddle point at the top of the barrier has $C_{2 v}$ symmetry. $C_{s}$ and $C_{2 v}$ are the names of point groups. Tunnelling splittings were the focus of several of the experimental studies. A number of papers used a 1-D model to convert an experimental tunnelling splitting into a barrier height. [5, 6, 8, 9] A 1-D model is accurate if it is possible to move from one equilibrium structure to the other by changing the value of a single coordinate and if that coordinate is weakly coupled to all other coordinates. For the VR this does not seem to be the case and therefore two groups have done full-dimensional variational calculations. [3, 4] They are only possible because two other groups have built a full-dimensional potential energy surface (PES). [1, 2]

VR has 5 atoms and therefore 9 vibrational coordinates and this by itself ensures that variational calculations are challenging. The most straightforward approach is to choose coordinates, use a direct product basis, and use an iterative eigensolver. [17-20] Each of the direct product basis functions is a product of functions of a single coordinate. For VR, direct-product discrete variable representation variational calculations have been done by S̆mydke et al.[4]. Calculations of this type are limited by the size of the vectors that must be stored in computer memory. [21] For a 9-D problem, if $n$ basis functions are required for each coordinate, the size of the vectors is $n^{9}$. Often $n \sim 10$ which means that one vector requires about $8 \mathrm{~GB}$ of memory.

In the VR case, variational calculations are especially difficult because it has two accessible wells. Although the (exact) kinetic energy operator is complicated, normal coordinates are good for the purpose of computing the (ro-)vibrational spectrum of many single-well molecules. For most multi-well molecules, normal coordinates are a poor choice. For VR, some sort of geometrically defined curvilinear internal coordinates are imperative. A harmonic basis is always poor for a multi-well molecule. For multi-well molecules a spherical harmonic type basis, with functions having amplitude everywhere in configuration space, is a good choice. A spherical harmonic type basis is a nondirect product basis and enables one to deal with unavoidable singularities in the KEO. It is also possible to use a related direct 
product (discrete variable representation) DVR basis[22], but is larger.

Rather than using basis functions that are products of 1-D factors, it is possible to use basis functions that are products of factors some of which are contracted functions depending on more than one coordinate. [23-31] For triatomic molecules, a nondirect product contracted basis was used many years ago by Bacic and Light.[23] For larger molecules, especially when using an iterative eigensolver, it is easier to use a direct product contracted basis. [32-34] The basis functions are products of factors that depend on a subset of the coordinates. The factors are obtained by solving reduced-dimension eigenproblems. In Yu's calculations only one factor is contracted. [3, 34-36] Due to the contraction(s), such calculations require less memory and less CPU time (if the contraction is reasonably efficient). Both S̆mydke et al.[4] and $\mathrm{Yu}$ et al. [3] have done contracted calculations for VR.

There are significant differences between the vibrational energy levels computed by S̆mydke et al.[4] and by $\mathrm{Yu}$ et al. [3] In this paper we present a third set of variational energy levels and discuss possible sources of the discrepancy between S̆mydke et al. and Yu et al. To do our calculation, we use a contracted basis method and demonstrate that it works well. We also comment on differences between our method and the contracted basis method of S̆mydke et al..

\section{COORDINATES, BASIS FUNCTIONS, AND HAMILTONIAN}

We use the polyspherical coordinates associated with the Radau-Jacobi vectors shown in Fig. 1. [37] The vectors are $\left(\mathbf{q}_{\mathbf{0}}, \mathbf{q}_{\mathbf{1}}, \mathbf{q}_{\mathbf{2}}, \mathbf{q}_{3}\right) \cdot \mathbf{q}_{\mathbf{2}}$ and $\mathbf{q}_{\mathbf{3}}$ are $1+2$ Radau vectors [38] for the $\beta-\mathrm{CH}_{2}$ group and $\mathbf{q}_{\mathbf{0}}$ and $\mathbf{q}_{\mathbf{1}}$ are Jacobi vectors. A mix of Radau and Jacobi vectors is sometimes advantageous. [17] The polyspherical angles are defined by attaching a body-fixed frame to a subset of the vectors. The $z$-axis is along $\mathbf{q}_{\mathbf{0}}$ and the $x$-axis is along $\mathbf{q}_{\mathbf{0}} \times \mathbf{q}_{\mathbf{1}} \times \mathbf{q}_{\mathbf{0}}$. We use the standard angles: $\theta_{1}$ is the angle between $\mathbf{q}_{\mathbf{0}}$ and $\mathbf{q}_{\mathbf{1}} ;\left(\theta_{i}, \phi_{i}\right)(i=2,3)$ are the spherical polar angles for $\mathbf{q}_{\mathbf{i}}$ in the above-mentioned frame. [37] To evaluate the potential at a particular Radau-Jacobi polyspherical point, we transform from the Cartesian coordinates of Radau-Jacobi vectors in an arbitrarily chosen frame, $\left(\mathbf{q}_{\mathbf{0}}, \mathbf{q}_{\mathbf{1}}, \mathbf{q}_{\mathbf{2}}, \mathbf{q}_{\mathbf{3}}\right)$ to Cartesian coordinates of bond vectors $\left(\mathbf{b}_{\mathbf{0}}, \mathbf{b}_{\mathbf{1}}, \mathbf{b}_{\mathbf{2}}, \mathbf{b}_{\mathbf{3}}\right)$ (defined in the caption of Fig. 1) in the same frame. This is 
done with the transformation matrix given below,

$$
\left(\begin{array}{l}
\mathbf{b}_{\mathbf{0}} \\
\mathbf{b}_{\mathbf{1}} \\
\mathbf{b}_{\mathbf{2}} \\
\mathbf{b}_{\mathbf{3}}
\end{array}\right)=\left(\begin{array}{cccc}
1-\frac{m_{1}}{m_{1}+m_{4}} & \frac{m_{2}}{\sqrt{\left(m_{2}+m_{3}+m_{5}\right) m_{5}}} & \frac{m_{3}}{\sqrt{\left(m_{2}+m_{3}+m_{5}\right) m_{5}}} \\
0 & 1 & 0 & 0 \\
0 & 0 & 1+\alpha_{523} m_{2} & \alpha_{523} m_{3} \\
0 & 0 & \alpha_{523} m_{2} & 1+\alpha_{523} m_{3}
\end{array}\right)\left(\begin{array}{c}
\mathbf{q}_{\mathbf{0}} \\
\mathbf{q}_{\mathbf{1}} \\
\mathbf{q}_{\mathbf{2}} \\
\mathbf{q}_{\mathbf{3}}
\end{array}\right),
$$

where

$$
\alpha_{523}=\frac{1}{m_{2}+m_{3}}\left(\sqrt{\frac{m_{2}+m_{3}+m_{5}}{m_{5}}-1}\right) .
$$

$m_{i}$ is the mass of atom $i$. Subsequently, the Cartesian coordinates of each atom are computed from the bond vectors and used to call the PES code.

As mentioned in the introduction, we use a product contracted (PC) basis. [33] Its functions, denoted $|b, s\rangle=|b\rangle|s\rangle$, are products of functions of the bend coordinates and functions of the stretch coordinates. The stretch and bend contracted functions are computed in primitive bases. For the stretch problem, the primitive basis functions are products of 4-D potential-optimized discrete variable representation (PODVR) functions [28]; for the bend problem, the primitive basis functions are products of two spherical harmonics, one for $\left(\theta_{2}, \phi_{2}\right)$ and one for $\left(\theta_{3}, \phi_{3}\right)$, and an associated Legendre function for $\theta_{1}$. We refer to this bend basis as a spherical harmonic type basis. Similar bend bases have been used in many calculations. [39-42] Because the frame is not attached to $\mathbf{q}_{\mathbf{2}}$ and $\mathbf{q}_{\mathbf{3}}$, it is straightforward to exploit symmetry. To do so one must apply the (23) permutation operator (see Fig. 1 for the atom labels). For the 5 -D bend basis this entails permuting $\left(l_{2}, m_{2}\right)$ with $\left(l_{3}, m_{3}\right)$.

The stretch, $|s\rangle$, and bend, $|b\rangle$, functions are eigenfunctions of stretch and bend reference Hamiltonians. In this paragraph we explain how the stretch and bend reference Hamiltonians are obtained from the full Hamiltonian. The vibrational Hamiltonian of an $N$-atom molecule in polyspherical coordinates $[37,43,44]$ associated with orthogonal vectors can be written,[45]

$$
\begin{aligned}
H_{v} & =T_{s}+\sum_{i=0}^{N-2} B_{i}\left(R_{i}\right) \hat{\mathcal{L}}_{i}(\theta)+V(R, \theta), \\
T_{s} & =-\sum_{i=0}^{N-2}\left(1 / 2 \mu_{i}\right)\left(\partial^{2} / \partial R_{i}^{2}\right), \\
B_{i}\left(R_{i}\right) & =1 /\left(2 \mu_{i} R_{i}^{2}\right) .
\end{aligned}
$$

$R_{i}$ are the lengths of the polyspherical vectors $\left[\left(\mathbf{q}_{\mathbf{0}}, \mathbf{q}_{\mathbf{1}}, \mathbf{q}_{\mathbf{2}}, \mathbf{q}_{\mathbf{3}}\right)\right.$ for $\left.\mathrm{VR}\right] ; T_{s}$ is the stretch kinetic energy operator (KEO); $\mu_{i}$ is the appropriate mass for $R_{i} ; \hat{\mathcal{L}}_{i}$ are angular operators, 
written in terms of vibrational angular momentum operators and operators that depend on $\theta_{1}[44]$; and $V$ is the potential energy function. $R$ without an index represents all the stretch coordinates and $\theta$ without an index represents all the bend coordinates. To define reference bend and stretch Hamiltonians, the vibrational Hamiltonian is divided as,

$$
H_{v}=H_{s}+H_{b}+\Delta T+\Delta V
$$

where,

$$
\begin{aligned}
H_{s} & =T_{s}+V_{\text {sref }}(R), \\
H_{b} & =\sum_{i=0}^{N-2} B_{i}\left(R_{i, e}\right) \hat{\mathcal{L}}_{i}(\theta)+V_{\text {bref }}(\theta), \\
\Delta T & =\sum_{i=0}^{N-2}\left[B_{i}\left(R_{i}\right)-B_{i}\left(R_{i, e}\right)\right] \hat{\mathcal{L}}_{i}(\theta), \\
\Delta V & =V(R, \theta)-V_{\text {bref }}(\theta)-V_{\text {sref }}(R) .
\end{aligned}
$$

$V_{\text {bref }}$ and $V_{\text {sref }}$ are reference potentials that one chooses. We compute eigenvalues of $H_{v}$ in a direct product basis each of whose functions is a product of an eigenfunction of $H_{s}$ and an eigenfunction of $H_{b}$. An eigenfunction of $H_{s}$ is denoted $|s\rangle$ and an an eigenfunction of $H_{b}$ is denoted $|b\rangle$. The eigenvalue problems for $H_{v}, H_{b}$, and $H_{s}$ are all solved using the Cullum and Willoughby Lanczos algorithm [46, 47]

For VR, we want $H_{s}$ and $H_{b}$ to be unchanged by (23) (permutation [48] of the $\mathrm{H}$ atoms of the $\beta-\mathrm{CH}_{2}$ group). When defining $H_{b}$, we therefore evaluate the the $B_{i}\left(R_{i}\right)$ coefficients at $R_{i}=R_{i, e}$, the stretch coordinate values at the saddle point. We also cut potentials through the saddle point structure to ensure that $H_{s}$ and $H_{b}$ are unchanged by (23). Hence, $V_{\text {bref }}(\theta)=V\left(R_{e}, \theta\right)$ and $V_{\text {sref }}(R)=V\left(R, \theta_{e}\right)$ where $R_{e}$ and $\theta_{e}$ are coordinate values at the saddle point, given in Table I.

For VR, Yu et al[3] used the same coordinates as we do. They also contracted the bend basis, but they did not contract the stretch basis. They called the contracted bend functions $|b\rangle$, vibrationally diabatic functions. They are actually crude adiabatic functions. Yu et al. solved the bend problem in a DVR basis whereas we use a spherical harmonic type basis and quadrature. Yu et al. used a slightly different definition of the bend reference Hamiltonian (see below). 
For VR, S̆mydke et al.[4] did variational calculations with the GENIUSH method. They use internal coordinates based on bond vectors. Their published results are obtained with a direct product PODVR basis, but they also discuss the use of a contracted basis. Their KEO has more terms, and the terms are more complicated, than our Radau-Jacobi KEO. Because they use a direct product DVR, it is easy to deal with coordinate dependent functions in terms of the KEO (G-matrix elements are computed at points). However, the cost of their calculation does depend on the number of terms and, owing to existence of KEO terms with both bend and stretch momenta, their KEO has more terms than the Radau-Jacobi KEO we use.

\section{SYMMETRY ERRORS AND HOLES ON THE NN-PES}

Yu et al. [3] used the original NN(neural network)-PES.[1] S̆mydke et al.[4] discovered that the original NN-PES, which we denote $V_{\mathrm{NN}}$, is not invariant with respect to (wrt) (23) ((23) permutes the two $\mathrm{H}$ atoms of $\beta-\mathrm{CH}_{2}$ group) and they modified it to make it invariant wrt (23) by averaging, see Eq. (A2) in the Appendix. We call the S̆mydke et al. PES $V_{\text {sym }}$. We find that $V_{\mathrm{NN}}$ is not invariant wrt (23) at points at which two bond lengths are equal, $R_{\mathrm{C}_{5}-\mathrm{H}_{2}}=R_{\mathrm{C}_{5}-\mathrm{H}_{3}}$; see the Appendix. Only the cut with $R_{\mathrm{C}_{5}-\mathrm{H}_{2}}=R_{\mathrm{C}_{5}-\mathrm{H}_{3}}$ is affected, but close to the most important tunnelling path the error is significant (see the Appendix). Errors are larger at higher potential values and they seem to have a bigger effect on higher rather than lower energy levels; see section VI. Low-lying levels seem not to be seriously influenced, perhaps because only a small region of the PES is affected.

Unfortunately, $V_{\mathrm{sym}}$ is not invariant wrt $E^{*}$ (the parity operator). This second type of symmetry error exists at some (not all) points with $R_{\mathrm{C}_{5}-\mathrm{H}_{2}}=R_{\mathrm{C}_{5}-\mathrm{H}_{3}}$. It is due to round-off error. The round-off error occurs when internal (Radau-Jacobi) coordinates are converted to Cartesian coordinates, which the PES routine requires. $V_{\text {sym }}$ inherits this error from $V_{\mathrm{NN}}$. The number of points on $V_{\text {sym }}$ affected by the parity symmetry error is smaller than the number of points on $V_{\mathrm{NN}}$ affected by the (23) symmetry error. Therefore, the parity error of $V_{\text {sym }}$ should have a small effect on energies. As explained in the Appendix, we fix the parity symmetry error of $V_{\mathrm{NN}}$ by changing a line of the original PES code before averaging. We

call our modified PES $\tilde{V}_{\text {sym }}$. $\tilde{V}_{\text {sym }}$ is used in our calculation and it is invariant wrt to (23) 
and $E^{*}$.

$V_{\mathrm{NN}}$ also has serious holes that must be dealt with to use it to compute vibrational energy levels with our method. Holes occur where the PES should have large values. They are due to the fact that there are too few ab initio points in these high-potential regions. On $V_{\mathrm{NN}}$, there are holes when the $\mathrm{H}$ of the top $\mathrm{CH}$ group is bent towards the lower $\mathrm{CH}_{2}$ group. See Fig. 1 of the supplementary material. We plug the hole by replacing potential values for points with $\angle \mathrm{C}_{5}-\mathrm{C}_{4}-\mathrm{H}_{1}$ in the range $\left[0^{\circ}, 90^{\circ}\right]$, corresponding roughly to $\left[\sim 90^{\circ}, \sim 180^{\circ}\right]$ for $\theta_{1}$, with $30000 \mathrm{~cm}^{-1}$. The high potential we impose prevents exchange of all three $\mathrm{H}$ atoms.

\section{CALCULATION DETAILS}

We use the PC basis mentioned in section II. Two sets of $|b\rangle$ are computed, one for each parity. The primitive bend basis functions are the parity adapted basis functions of Ref. 44 . As explained in section II, the reference configuration used to define $H_{b}$ is defined so that the $|b\rangle$ functions are either symmetric or antisymmetric wrt (23). This makes it possible to calculate symmetric and antisymmetric $|b\rangle$ separately, which speeds up their calculation. It also has the advantage that $|b\rangle$ functions have symmetry labels. Because they have symmetry labels, the size of the final PC basis can be reduced by a factor of two. It would be possible to calculate both symmetric and antisymmetric $|b\rangle$ from a single set of matrix-vector products (MVPs) using the symmetry adapted Lanczos algorithm. [49, 50] Instead, we calculate them separately, but in parallel, by using projection operators. The projection operator is applied to Lanczos vectors $v_{1+8 i}$ and $v_{2+8 i} i=0,1,2, \cdots$. [51]

Yu et al [3] also contract the bend basis, they also use a $V_{\text {bref }}$ that is invariant wrt (23), but theirs is obtained by fixing the Radau-Jacobi lengths $R_{0}$ and $R_{1}$ to equilibrium values and finding values of the Radau lengths $R_{2}=R_{3}$ that minimize the potential. Our $V_{\text {bref }}$ is flatter and therefore their $|b\rangle$ may be better basis functions for describing wavefunctions localized in the two wells. Because we use thousands of bend functions, the difference between the two $V_{\text {bref }}$ should not be important.

The basis functions used in our calculation are summarized in Table II. The maximum values of the labels of spherical harmonics type primitive bend basis are: $l_{i x}=m_{i x}=29$ with $l_{i} \leq l_{i x},\left|m_{i}\right| \leq m_{i x}$ and $l_{i} \geq\left|m_{i}\right|$ for $i=1,2$ and 3 . We use two sets of contracted 
bend functions to test the convergence, basis b1 and b2. Basis b1 (b2) has $N_{b}=4038(5780)$ (including both even and odd parity) contracted bend functions. We use only one set of contracted stretch functions, since the cut-off energy $E_{s}^{\text {cut }}=20000 \mathrm{~cm}^{-1}$ is high. We compare levels computed with b1s1 and b2s1 and determine that levels up to $3000 \mathrm{~cm}^{-1}$ are converged to about $0.001 \mathrm{~cm}^{-1}$. We use the smaller b1s1 basis to compute the levels and wavefunctions reported in the tables of this paper.

The only non-obvious part of a calculation with a $\mathrm{PC}$ basis is dealing with $\Delta T$ and $\Delta V$ (see Eq. (4)). Eigenfunctions of $H_{b}$ and $H_{s}$ are computed using the same procedure that is used to solve the Schroedinger equation without contracted basis functions. [33, 39] If the $\mathrm{PC}$ basis is small enough, then it is possible to compute matrix elements of $\Delta T$ and $\Delta V$ and explicitly diagonalize $H_{v}$. $[4,30,31]$ On the other hand, if the PC basis is large, then it is better to solve the final matrix eigenvalue problem with an iterative algorithm. This can be done, without computing matrix elements of $\Delta T$ and $\Delta V$, by evaluating sums sequentially. [33] Matrix-vector products with $\Delta V$ are the most difficult. Because we are using a general PES, we need quadrature. The simplest thing to do would be to transform each (Lanczos) vector to which we apply $\Delta V$ from the basis to the quadrature grid and then back to the basis. This would require storing a vector as large as the size of the full quadrature grid. Instead, we use the $\mathbf{F}$ matrix idea that reduces both the required $\mathrm{CPU}$ time and the required memory[33]. An element of the $\mathbf{F}$ matrix is $\left\langle b^{\prime}\left|\Delta V\left(r_{\alpha}, \theta\right)\right| b\right\rangle$. Computing the $\mathbf{F}$ matrix is costly. Fortunately, its computation can be trivially parallelized and programmed with MPI. $[39,51]$ Each MPI process handles a set of sub-matrices each of which has rows and columns labelled by a subset of the $N_{b}|b\rangle$ functions. A master process sends potential values for a stretch DVR point simultaneously to all the MPI processes. This is the only communication between processors and the amount of data sent is minimal. The parallelization efficiency is therefore nearly $100 \%$. We have also utilized the (23) permutation symmetry to reduce the memory required to store $\mathbf{F}[52,53]$ by exploiting the fact that the entire $\mathbf{F}$ can be computed from its elements at only 3158 DVR points. Without this trick we would need to store $\mathbf{F}$ elements at 5711 points; See Table II. For the b2s1 basis, the $\mathbf{F}$ matrix calculation takes 3 hours with 360 cores (on a computer with 9 40-core Xeon processors). This time could be reduced by using more cores. As explained previously, we need a set of $|b\rangle$ functions for each parity. There are therefore two $\mathbf{F}$ matrices, but they can be computed independently. For 
the b2s1 basis, the even and odd $\mathbf{F}$ matrix sizes are 49 GB and 44 GB. In the final Lanczos calculation in the $|b s\rangle$ basis, one matrix-vector product takes about 8 seconds on one Xeon CPU with 40 cores, using OPENMP parallelization.

\section{ASSIGNMENT OF CALCULATED LEVELS}

In sections III and IV, we explain how we compute VR energy levels. To understand our results we must assign them. We do this by using energy level patterns and wavefunctions. It is straightforward to use Lanczos to calculate eigenvalues. [46] It is harder to calculate eigenvectors which are of course needed to get wavefunctions. It is necessary to carefully select the number of Lanczos vectors to combine to make an eigenvector. [32, 39, 46] To use wavefunctions to assign, one can plot either wavefunction cuts or probability densities. [54] Some authors refer to probability densities as reduced density matrices. [55] Because probability densities are obtained by integrating over some of the coordinates, it is in some cases better to use wavefunction cuts. [56, 57]

Most of the VR states we calculate have one dominant $|b s\rangle$ component. Normal mode labels can be linked to these states by assigning normal mode labels to the $|b\rangle$ and $|s\rangle$ functions. $|s\rangle$ functions can be simply assigned using their energies. We assign $|b\rangle$ functions by plotting cuts through $|b\rangle$, obtained by fixing all but two of the polyspherical angles used to calculate the spectrum to saddle-point values. To make $|b\rangle$ plots, one needs to be able to evaluate the bend basis functions at arbitrary points. This we can do because we have analytic expressions for the bend basis functions. [44, 58] In a previous paper, [44] we plotted $|b\rangle$ as functions of a pair of symmetry coordinates.

When analysing the cuts, we look for motion along the normal coordinates. We use $\mathrm{Yu}$ et al. [3]'s description of the 9 normal modes. There are four stretch modes: $\nu_{1}$ is the $\mathrm{C}_{\alpha} \mathrm{H}$ stretch mode; $\nu_{2}$ and $\nu_{3}$ are the antisymmetric and symmetric $\mathrm{CH}$ stretch modes of $\mathrm{CH}_{2}$, respectively; $\nu_{4}$ is the $\mathrm{CC}$ stretch mode. There are five bend modes: $\nu_{5}$ is the $\mathrm{CH}_{2}$ bend mode; $\nu_{6}$ and $\nu_{7}$ are the trans-HCCH and cis- $\mathrm{HCCH}$ bend modes, respectively; $\nu_{8}$ is the $\mathrm{CH}_{2}$ out-of-plane wagging mode. $\nu_{9}$ is the $\mathrm{C}_{\alpha} \mathrm{H}$ out-of-plane internal rotation (similar to the $\mathrm{OH}$ internal rotation of methanol). Although the plots are functions of the polyspherical angles and not bond angles, it is possible to recognize motion along normal coordinates because 
the Radau-Jacobi angles are similar to bond angles.

Increasing the $q_{\nu_{5}}$ normal coordinate corresponds to increasing both $\theta_{2}$ and $\theta_{3}$. The $\nu_{5}$ and $2 \nu_{5}$ states therefore have node(s) along the line $\theta_{3}=\theta_{2}$; see Fig. 2. Increasing the $q_{\nu_{8}}$ normal coordinate corresponds to increasing $\phi_{2}$ and decreasing $\phi_{3}$. In Fig. 3, the wavefunctions localized in the well at $\left(\phi_{2}, \phi_{3}\right)=(0, \pi)$ and in the well at $\left(\phi_{2}, \phi_{3}\right)=(\pi, 2 \pi)$ both have node(s) along lines with negative slope. In Fig. 3, because the wells are centered at $\phi_{i}=0$ or $2 \pi$, and therefore at the edges of the plot, the wavefunctions are split into two parts. The two parts are actually connected because the wavefunctions are periodic in $\phi_{i}$ with a period of $2 \pi$. Increasing the $q_{\nu_{9}}$ normal coordinate corresponds to increasing both $\phi_{2}$ and $\phi_{3}$. In Fig. 4, the wavefunctions have node(s) along lines with positive slope. In Fig. 4 panel (a), there is a node between the positive lobe near $\left(\phi_{2}, \phi_{3}\right)=(0, \pi)$ and the negative lobe near $\left(\phi_{2}, \phi_{3}\right)=(2 \pi, \pi)$ (both in the same well). In Fig. 4 panels (c) and (d), it is clear that wavefunctions are de-localized over both wells. Both the out-of-plane modes are very clean and we can easily assign states with as many as 4 quanta of excitation.

$\nu_{6}$ and $\nu_{7}$ states are harder to assign because the corresponding (in-plane) coordinates are strongly coupled. When the states are plotted as functions of $\theta_{1}, \theta_{3}$, the $\nu_{6}$ and $\nu_{7}$ states have nodes along $\theta_{1}-\theta_{3}$ and $\theta_{1}+\theta_{3}$, respectively. See Fig. 5. These are consistent with the normal mode arrows of the trans-HCCH and cis- $\mathrm{HCCH}$ modes shown in Ref. 3. When moving along the $\nu_{7}$ coordinate, $\theta_{1}$ changes more than $\theta_{3}$. More excited $\nu_{6}$ and $\nu_{7}$ states become less regular, due to coupling, and it is not possible to identify lines along which there is nodal structure. In principle, it might be possible to assign $\nu_{6}$ and $\nu_{7}$ states using a plot of wavefunctions as functions of $\left(\theta_{2}, \theta_{3}\right)$. However, we find that this does not provide information that cannot be extracted from the $\left(\theta_{1}, \theta_{3}\right)$ plots.

In previous papers on VR, different ideas are used to make assignments. Yu et al.[3] assigned their levels by computing the expectation value of the square of normal coordinates and using the Hellmann-Feynman theorem. S̆mydke et al. [4] assigned their levels by computing probability densities as functions of pairs of coordinates. They are obtained by integrating squared wavefunctions over all the remaining coordinates. 


\section{RESULTS AND COMPARISON WITH THE TWO PREVIOUS VARIA- TIONAL CALCULATIONS}

Our calculated vibrational levels of VR are given in Tables III, IV, V and VI for each of the four $G_{4}$ symmetries. In these tables we also compare with the previous two calculations using related PESs. We compare by listing, for all three calculations, energy levels in order of increasing energy. This is an informative way to compare different calculations if they are done on the same PES and are very accurate. If these conditions are not satisfied, then even the number of levels below some threshold may not be the same for the two calculations. In this case, if it is possible to make reliable assignments, then it is better not to compare the $n$th level from one ordered list with the $n$th level from another ordered list, but to compare levels with the same assignment. Ordering the levels and comparing the $n$th levels has the advantage that it does not depend on the correctness of assignments. Assignments are less meaningful and harder to determine at high energies. However, for VR some of the highlying states can be assigned unambiguously and in these cases we compare levels with the same assignment.

There are significant differences between the three sets of VR energy levels. For example, even the number of levels below the lowest assigned high-lying $\mathrm{CH}$ stretch level is different. $\breve{S}$ mydke et al. compared their levels with those of Yu et al. by matching assignments. They reported significant differences (as large as $230 \mathrm{~cm}^{-1}$ ) between their assigned levels and $\mathrm{Yu}$ et al.'s assigned levels. In some cases the differences are much less if the matching is done by energy ordering. S̆mydke et al. cite two reasons for the differences. First, they used an averaged NN-PES (see the discussion of $V_{\text {sym }}$ in the appendix), whereas $\mathrm{Yu}$ et al. used the original NN-PES ( $V_{\mathrm{NN}}$ in the appendix). Second, they suggested that Yu et al.'s levels are not converged because $\mathrm{Yu}$ et al. used only $500|b\rangle$ for both parities together. [59] We check Yu et al.'s convergence error by doing calculations with two different bend basis sizes: $N_{b}=507$ and $N_{b}=5586$ (for both parities together). These two bases are obtained with $E_{b}^{\text {cut }}=5080 \mathrm{~cm}^{-1}$ and $E_{b}^{\text {cut }}=9800 \mathrm{~cm}^{-1}$. The smaller basis roughly corresponds to the basis of $\mathrm{Yu}$ et al. . We find that for levels up to $3040 \mathrm{~cm}^{-1}$, convergence errors with the smaller basis are less than $3 \mathrm{~cm}^{-1}$ and for levels up to $2500 \mathrm{~cm}^{-1}$ they are less than $0.5 \mathrm{~cm}^{-1}$. Yu et al.'s convergence errors must be similar and therefore convergence error is probably not 
responsible for the differences observed by S̆mydke et al. S̆mydke et al. stated that their levels up to $3200 \mathrm{~cm}^{-1}$ are converged to within $0.05 \mathrm{~cm}^{-1}$. If the levels of S̆mydke et al. and of $\mathrm{Yu}$ et al. are well converged then differences between their levels must be due to something else.

To compare the three calculations, we shall focus on levels with $A+$ symmetry. Below about $2500 \mathrm{~cm}^{-1}$ (above the black line in Table III), our levels agree rather well with those of Yu et al., with differences on the order of $1 \mathrm{~cm}^{-1}$, and agree less well the levels of S̆mydke et al., with differences as large as $28 \mathrm{~cm}^{-1}$. In this region, the three sets of assignments are identical. The comparison is slightly complicated by the fact that the three calculations use three different PESs. Yu et al. use $V_{\mathrm{NN}}$; S̆ Smydke et al. use $V_{\text {sym }}$; we use $\tilde{V}_{\text {sym }}$ (see the appendix). The good agreement between our levels and Yu et al.'s levels implies that the non-invariance of $V_{\mathrm{NN}}$ wrt $(23)$ and $E^{*}$ does not significantly affect low-lying levels. This is not surprising because the non-invariance errors of $V_{\mathrm{NN}}$ only occur at the small set of points with $R_{\mathrm{C}_{5}-\mathrm{H}_{2}}=R_{\mathrm{C}_{5}-\mathrm{H}_{3}}$. $V_{\text {sym }}$ and $\tilde{V}_{\text {sym }}$ differ at an even smaller set of points (about one third smaller, see the appendix). The magnitude of the differences is also smaller. For these reasons, we expect differences between $V_{\text {sym }}$ and $\tilde{V}_{\text {sym }}$ not to significantly affect energy levels. The disagreement between our levels and those of S̆mydke et al. suggests that something else may be affecting the accuracy of the S̆mydke et al. calculation.

Above about $2500 \mathrm{~cm}^{-1}$, our levels agree poorly with both those of Yu et al. and those of S̆mydke et al. We believe that the discrepancy between our and Yu et al.'s levels in this energy range is due to differences between $V_{\mathrm{NN}}$ and $\tilde{V}_{\text {sym }}$ at points with $R_{\mathrm{C}_{5}-\mathrm{H}_{2}}=R_{\mathrm{C}_{5}-\mathrm{H}_{3}}$. These differences are much larger at higher energies. At the $4 \nu_{7}\left(\nu_{7}\right.$ the cis-HCCH bending mode) level at $2555 \mathrm{~cm}^{-1}$, the difference between the levels suddenly jumps to $16 \mathrm{~cm}^{-1}$.

Because of the large differences between the three calculations above $2500 \mathrm{~cm}^{-1}$, comparing levels in this energy region in energy increasing order is no longer the best choice. If we compare levels with the same assignments when the assignments are certain, the differences between our levels and S̆mydke et al.'s and Yu et al.'s levels are actually smaller than the differences given in Tables III to VI. This is shown in Table VII where we select some levels above $2500 \mathrm{~cm}^{-1}$ to compare. For example, the $\nu_{1}$ levels of all three calculations agree to within $1 \mathrm{~cm}^{-1}$ (See Table VII). These $\nu_{1}$ levels are the 43rd, 44th, and 42nd levels in Cal1, Cal2 and Cal3. When comparison is made on the basis of assignment, S̆mydke et al.'s stretch 
levels are in good agreement with our levels (to within $0.5 \mathrm{~cm}^{-1}$ ) and to a lesser extent in agreement with $\mathrm{Yu}$ et al. 's levels (to within $4.4 \mathrm{~cm}^{-1}$ ). This suggests that there may be a problem with the bend part of S̆mydke et al.'s calculation.

It seems unlikely that the difference between $V_{\text {sym }}$ and our $\tilde{V}_{\text {sym }}$ is responsible for differences between our levels and those of S̆mydke et al. It is possible that all the differences are due to convergence errors. In their bend calculation, S̆mydke et al. define their five angles $\vartheta_{1}, \vartheta_{2}, \vartheta_{3}, \varphi_{1}, \varphi_{2}$ in their Figure 2, but not using equations. The top green plane in their Fig. 2 contains only one $\mathrm{C}_{\alpha}-\mathrm{H}$ vector. It is actually defined as the plane that contains that $\mathrm{C}_{\alpha}-\mathrm{H}$ vector and the blue line that is the intersection between the bottom green plane containing $\mathrm{CH}_{2}$ and the plane that is perpendicular to the vector along $\mathrm{C}_{1}-\mathrm{C}_{2}[60]$.

$\breve{S}$ mydke et al. reduce the range of their coordinates and correspondingly scale their DVR points. For $\vartheta_{1}$ and $\vartheta_{2}$, the range is reduced from $[0, \pi]$ to about $[0, \pi / 2]$. For $\varphi_{1}$ and $\varphi_{2}$ the range is reduced from $[0,2 \pi]$ to about $[0, \pi]$. In total they reduce the 5 -D bend configuration space by a factor of 16 . This reduction will introduce no error, only if the potential is large in the excluded region. Rather than restricting coordinate ranges to avoid points with high potential values, we use an FBR (finite basis representation) bend basis whose functions have amplitude everywhere in configuration space and replace potential values larger than a ceiling value with the ceiling value. This ceiling reduces the number of required MVPs.[61] It also decreases the memory cost of storing wavefunctions on the quadrature grid [39] and speeds up the evaluation of MVPs because one can ignore elements of grid vectors whose potential values are above the ceiling $[62,63]$. We choose a ceiling value of $V_{b}^{\text {ceil }}=22180$ $\mathrm{cm}^{-1}$. The same value was used to prune the DVR basis used to compute $|b\rangle$ by Yu et al. [3]. The value is high enough given that the energy levels we want to compute are below 4000 $\mathrm{cm}^{-1}$. In the 5-D bend space, we need to keep only the $N_{\text {quad }}$ points that are under the ceiling value. See Table II for $N_{\text {quad }}$. About $7.5 \%$ of quadrature points are under the ceiling value. Even though the context is different, this ratio may be compared to the factor of 16 reduction used in S̆mydke et al.'s calculation with a DVR basis. The ceiling does not reduce the FBR basis size. We also reduce the range of our $\phi_{2}$ quadrature points (alternatively we could also choose to reduce the range for $\phi_{3}$ ) from $[0,2 \pi]$ to $[0, \pi]$ because we exploit parity symmetry. This reduction introduces no error regardless of PES values. We are implicitly using all the $\phi_{2}$ and $\phi_{3}$ points. 
S̆mydke et al. also reported a calculation using a contracted basis. The cost of their contracted-basis calculation is comparable to the cost of their direct-product calculation. As is the case in our calculation, their contracted functions are products of 4-D contracted stretch functions and 5-D contracted bend functions. With $N_{b}=501$ and $N_{s}=26$, they find that levels up to about $1500 \mathrm{~cm}^{-1}$ above the ZPE have errors less than $1 \mathrm{~cm}^{-1}$, but higher levels have larger errors, e.g. $8 \mathrm{~cm}^{-1}$ errors for levels near $3000 \mathrm{~cm}^{-1}$. In contrast, we are able to converge all levels up to $3000 \mathrm{~cm}^{-1}$ to about $0.001 \mathrm{~cm}^{-1}$ because we use a much larger bend basis (our $N_{b}=2858$ for one parity or $N_{b}=5586$ for two parities and our $N_{s}=$ $460)$.

There are several important differences between the contracted calculation of S̆mydke et al. and our contracted calculation that might explain why we are able to use much larger bases. First, the memory cost of their calculation is higher. We do not store a $N_{s} N_{b} \times N_{s} N_{b}$ matrix representing the KEO in the $|s b\rangle$ basis. Because we use polyspherical coordinates, each term in our $\Delta T$ operator is a product of stretch functions and bend operators. We need to store only $N_{b} \times N_{b}$ matrices representing the $\hat{\mathcal{L}}_{i}(\theta)$ operators in Eq. (5). S̆mydke et al. compute and store matrices in the $|b s\rangle$ basis for each term in their KEO. Due to their choice of bond coordinates they have many terms. Bond coordinates are not always bad, for some molecules the basis required to achieve convergence will be smaller when using bond coordinates. GENUISH has the advantage that it is simple to use even when bond coordinates are used. SMmydke et al. also store a matrix representing the PES in the $|b s\rangle$ basis. Instead, we store the $\mathbf{F}$ matrix. The ratio of the memory cost of storing the $\mathbf{V}$ matrix to the memory cost of storing the $\mathbf{F}$ matrix is proportional to $N_{s}^{2} / N_{\alpha}$. Therefore, the $\mathbf{F}$ matrix requires less memory than the $\mathbf{V}$ matrix, if $N_{s}^{2} / N_{\alpha}$ is larger than one. $N_{\alpha}$ is the number of DVR functions used to compute $|s\rangle . N_{\alpha}$ is called $N_{\text {DVR }}$ in Table II. In our VR calculation, $N_{s}^{2} / N_{\alpha}=460^{2} / 5711 \approx 37$ (these numbers do not take into account the (23) permutation symmetry). S̆mydke et al.'s $N_{s}$ is small enough that $N_{s}^{2} / N_{\alpha}<1$ and therefore the $\mathbf{F}$ matrix we use in this paper would not reduce their memory cost. However, by interchanging the role of bend and stretch one can define an interchanged $\mathbf{F}$ matrix (similar to what we did for $\mathrm{H}_{2} \mathrm{O}$ dimer [64] ) and it would reduce their memory cost. The interchanged $\mathbf{F}$ matrix is advantageous if $N_{b}^{2} / N_{\beta}>1 . N_{\beta}$ is the number of DVR functions used to compute $|b\rangle$

Second, S̆mydke et al. explicitly diagonalize a Hamiltonian matrix in the PC basis. 
The cost of this diagonalization scales as $N_{b}^{3} N_{s}^{3}$. On the other hand, we use an iterative eigensolver whose cost scales as $M N_{\alpha} N_{b}^{2}$, where $M$ is the number of required iterations and $N_{\alpha} N_{b}^{2}$ is the cost of a MVP (the potential MVP is the most costly). [33] Third, S̆mydke et al. compute $\mathbf{V}$ and we compute $\mathbf{F}$. The cost of computing $\mathbf{V}$ depends on how it is done. One efficient way to compute $\mathbf{V}$ is from the interchanged $\mathbf{F}$. [65] It is clearly more costly to compute $\mathbf{V}$ than the interchanged $\mathbf{F}$. Fourth, we reduce the cost by discarding both bend and stretch points at high energies. [39] Fifth, The parity adapted basis we use reduces both the memory and the CPU cost and we take advantage of both parity and (23) permutation symmetry to reduce the cost of solving the eigenvalue problem in the PC basis.

\section{AN OUT-OF-PLANE $\mathrm{C}_{\alpha}$-H TUNNELLING PATH}

Tunnelling of the $\mathrm{H}$ atom of the $\mathrm{C}_{\alpha}-\mathrm{H}$ group is generally assumed to occur along the inplane $\mathrm{C}_{\alpha}-\mathrm{H}$ rocking path ( $\theta_{1}$ coordinate) through the saddle point $[3,4]$. The barrier height on the NN-PES is $1743 \mathrm{~cm}^{-1}$ (see Table I). Vibrational excitation of both the $\nu_{6}$ and $\nu_{7}$ modes facilitate this tunnelling motion (see e.g. Fig. 5) since the corresponding coordinates involve motion along $\theta_{1}$. This is borne out by the significantly enhanced tunnelling splittings of the corresponding fundamentals (about $14 \mathrm{~cm}^{-1}$ ), compared to those other vibrational modes. There is a secondary out-of-plane tunnelling path along the $\nu_{9}$ vibrational coordinate. It is corresponds to internal rotation of $\mathrm{C}_{\alpha}-\mathrm{H}$. This path was not previously discussed in the literature. To visualise wavefunctions along both the in-plane and out-of-plane paths, we define a new pair of spherical polar angles $(\tilde{\theta}, \tilde{\phi})$, describing the orientation of $\mathrm{H}_{\alpha}$ in a frame that is attached to the $\mathrm{C}_{\beta} \mathrm{H}_{2}$ group. The frame's $z$-axis is along $\mathbf{q}_{\mathbf{0}}$ and its $x$-axis is along $\mathbf{q}_{\mathbf{0}} \times \mathbf{q}_{\mathbf{2}} \times \mathbf{q}_{\mathbf{0}}$. To assess the relative importance of the two tunnelling paths, we plot 2-D $(\tilde{\theta}, \tilde{\phi})$ cuts of 5 -D bend wavefunctions obtained on $V_{\text {bref }}$. For these plots, $\theta_{1}=\tilde{\theta}, \phi_{2}=-\tilde{\phi}$, $\phi_{3}=\phi_{2}+\pi$, and $\theta_{2}$ and $\theta_{3}$ are fixed at their saddle point value of $120^{\circ}$. It would also be possible to convert $(\tilde{\theta}, \tilde{\phi})$ into Cartesian coordinates in the body-fixed frame defined above and to plot the $\mathrm{H}_{\alpha}$ atom density in the space. For a given state, if the density is high along a particular path then that path is an important tunnelling path. In Fig. 6(a), we show a potential cut of $V_{\text {bref }}$ as a function of $(\tilde{\theta}, \tilde{\phi})$. It helps us to understand the wavefunction cuts. The minimum for the potential cut is at $114 \mathrm{~cm}^{-1}$ with $(\tilde{\theta}, \tilde{\phi})=\left(38.5^{\circ}, 0^{\circ}\right)$ or $\left(38.5^{\circ}, 180\right)$, 
it is similar to the minimum on $V_{\text {bref }}$, and higher than global minimum (which is zero) on the 9-D PES because the stretch coordinates are fixed at the saddle point values. Fig. 6(c) clearly shows that maxima of the $4 \nu_{7}(A+)$ wavefunction are along the in-plane path that passes through the saddle point barrier of $1743 \mathrm{~cm}^{-1}$ and therefore that tunnelling is along this path. This is because the $4 \nu_{7}(A+)$ coordinate facilitates the tunnelling along the inplane path. Fig. 6(d) clearly shows that maxima of the $4 \nu_{9}(A+)$ wavefunction are along the out-of-plane path that corresponds roughly to movement along $\tilde{\phi}$, the internal rotation coordinate of $\mathrm{C}_{\alpha}-\mathrm{H}$ and therefore that tunnelling is along this path. This vertical path from $\mathrm{X}$ to $\mathrm{Y}$ with $\tilde{\theta}=38.5^{\circ}$ cuts across a bump. The maximum on the path is at $\tilde{\phi}=90^{\circ}$, where it is $3990 \mathrm{~cm}^{-1}$. The top of the bump is lowest at $\tilde{\theta}=0^{\circ}$ which is the saddle point with energy $1743 \mathrm{~cm}^{-1}$ The wavefunction amplitude at the top of the out-of-plane path barrier where $\tilde{\phi}=90^{\circ}$ is larger than the amplitude at the saddle point even though its potential energy is much lower. Note that even though we are using Radau-Jacobi coordinates, the above conclusions should be valid also for bond angles. They should also be valid for full 9-D wavefunctions because the 9-D wavefunctions are dominated by the 5 -D wavefunctions we plotted. The $(\tilde{\theta}, \tilde{\phi})$ plot has the additional advantage that it helps to assign $n \nu_{7}$ and $n \nu_{6}$ states, for example, the $3 \nu_{7}$ and $4 \nu_{7}$ states. Both states were not assigned by S̆mydke et al.

\section{COMPARISON WITH EXPERIMENTAL DATA}

Both Yu et al. and S̆mydke et al. summarize the available experimental VR data and compare them with their computed energy levels. Yu et al. identified a number of missassignments in the experimental papers. Since the NN-PES symmetry errors have a small effect on Yu et al.'s levels below $2500 \mathrm{~cm}^{-1}$, the miss-assignments they identify in this energy region remain valid. The fundamental vibrational levels on the NN-PES are quite accurate.

Every vibrational state is split by tunnelling between the two equivalent wells. The ground state is split into a lower $A+$ state and an upper $B+$ state because the $\mathrm{C}_{\alpha} \mathrm{H}$ tunnelling coordinate is $B+$ symmetry. $A+$ and $B+$ are labels of the $G_{4}$ permutation inversion group. $G_{4}$ is isomorphic with the $C_{2 v}$ point group, used by other researchers. Odd-parity vibrational states such as the $\nu_{8}$ and $\nu_{9}$ states are split into lower $A$ - and upper $B$ - components. Allowed transitions are between the lower tunnelling components and between the upper 
tunnelling components of either different or the same vibrational states. We denote the tunnelling splitting of the $\nu$ fundamental as $\Delta \nu$; it is defined as $E_{B+}-E_{A+}$ (for even-parity states) and $E_{B-}-E_{A-}$ (for odd-parity states). In most cases, the tunnelling splitting is positive, but it is negative for some states, due to coupling. In Table VIII, we compare our tunnelling splittings for the fundamentals with those of Šmydke et al. and Yu et al.

Experimentally measured tunnelling splittings are available for only three states : the ground state, $\nu_{8}$, and $\nu_{3}$. The three calculations agree well for the ground state and the $\nu_{8}$ tunnelling splittings. There is also agreement with experimental results for these two states, confirming the accuracy of the NN-PES. $\nu_{3}$ is the $\mathrm{CH}_{2}$ symmetric stretch state and its energy (near $2900 \mathrm{~cm}^{-1}$ ) is much higher than that of the $\nu_{8}$ state $\left(\right.$ near $890 \mathrm{~cm}^{-1}$ ). The NN-PES is less accurate for the $\nu_{3}$ tunnelling splitting. Dong et al.[10] observed the lower-to-lower and higher-to-higher $\nu_{3}$ bands of jet-cooled VR at high resolution. They determined the tunnelling splitting of $\nu_{3}$ to be $0.6144 \mathrm{~cm}^{-1}$. The slight increase of the tunnelling splitting, compared to the ground state value of $0.5428 \mathrm{~cm}^{-1}$, was explained as being due to lowering of the effective tunnelling barrier [10]. For this state, our calculated tunnelling splitting is 0.05, Yu et al.'s is 0.19 and S̆mydke et al.'s is $-1.33 \mathrm{~cm}^{-1}$. Clearly, for this state the splitting on the NN-PES is less accurate. Note that the higher experimental[10] tunnelling level for $\nu_{3}$ was quoted as $2901.9319 \mathrm{~cm}^{-1}$ in Table III of Ref. 3. This energy is relative to the higher tunneling level of the ground state. The correct number is $2902.4747 \mathrm{~cm}^{-1}$ by adding 0.5428 $\mathrm{cm}^{-1}$, the ground state tunneling splitting.

From assigned $n \nu_{7}$ states, we determine tunnelling splittings for $\nu_{7}, 2 \nu_{7}, 3 \nu_{7}$, and $4 \nu_{7}$. They are 13.9, 109.2, 191.9, 160.8 or $190.4 \mathrm{~cm}^{-1}$ (because there are two candidates for $\left.4 \nu_{7}(B+)\right)$. For higher overtones of $\nu_{7}$, the tunnelling splittings are large and irregular because the energies are above the barrier of the in-plane tunnelling path. We cannot extract tunnelling splittings for high overtones of $\nu_{6}$ states because we can not assign $2 \nu_{6}(B+)$ and higher $n \nu_{6}$ states. S̆mydke et al. also do not assign these states. The tunnelling splittings for $\nu_{9}, 2 \nu_{9}, 3 \nu_{9}$, and $4 \nu_{9}$ are $1.5(1.5), 3.4(3.6), 5.1(5.2), 9.8(6.4) \mathrm{cm}^{-1}$. The numbers in parentheses are those computed by S̆mydke et al. The energy for $4 \nu_{9}$ is $2959 \mathrm{~cm}^{-1}$, just below the out-of-plane path barrier on $V_{\text {bref }}, 3990 \mathrm{~cm}^{-1}$. The tunnelling splittings for $\nu_{8}, 2 \nu_{8}$, $3 \nu_{8}$, and $4 \nu_{8}$ are $0.6(0.6), 1.3(1.3), 2.1(2.9), 3.5$ (n.a.) $\mathrm{cm}^{-1}$. The numbers in parentheses are computed by S̆mydke et al.. They do not assign $4 \nu_{8}$. The energy of the $4 \nu_{8}$ state is 
$3568 \mathrm{~cm}^{-1}$. Even though this energy is much higher than the energy of the $4 \nu_{9}$ state, the tunnelling splitting is smaller than that of the $4 \nu_{9}$ state because $\nu_{8}$ is $\mathrm{CH}_{2}$ wagging which does not facilitate tunnelling along the out-of-plane path.

There are some experimentally observed splittings that are not discussed by Yu et al. and $\breve{S}$ mydke et al. Dong et al. observed a band they assigned to the lower-to-lower transition of the $\nu_{7} \rightarrow \nu_{3}+\nu_{7}\left(\nu_{7}\right.$ is an in-plane bend $)$ hot band. The band center is $4.63 \mathrm{~cm}^{-1}$ lower than the lower-to-lower $\nu_{3}$ band center. To compare with this experimental result, we assign $\nu_{3}+\nu_{7}(A+/ B+)$ levels. They were not assigned in Refs. 3, 4. The lower-to-lower band centre, $E_{A+}\left(\nu_{3}+\nu_{7}\right)-E_{A+}\left(\nu_{7}\right)$ is at $3563.56-667.66=2895.90 \mathrm{~cm}^{-1}$ (Table III) and the higher-to-higher band centre $E_{B+}\left(\nu_{3}+\nu_{7}\right)-E_{B+}\left(\nu_{7}\right)$ is at $3574.46-681.56=2892.90$ $\mathrm{cm}^{-1}$ (Table IV). The lower-to-lower band is red-shifted from the lower-to-lower $\nu_{3}$ band at $2900.37 \mathrm{~cm}^{-1}$ by $4.47 \mathrm{~cm}^{-1}$ (all calculated values). This red shift is in good agreement with the experimental shift of $4.63 \mathrm{~cm}^{-1}$. The higher-to-higher band is red-shifted from the $\nu_{3}$ band by $7.47 \mathrm{~cm}^{-1}$. Dong et al. observed only the lower-to-lower band and conjectured that the higher-to-higher band is substantially blue shifted, assuming that "vibrational excitation along the tunnelling coordinate should also increase the tunnelling splitting dramatically". Motion along the normal coordinate that corresponds to $\nu_{7}$ brings the molecule from one minimum towards the other minimum and the tunnelling splitting of $\nu_{7}\left(13.90 \mathrm{~cm}^{-1}\right)$ is indeed much larger than that of the ground state. Nonetheless, the tunnelling splitting for $\nu_{3}+\nu_{7}$ is $10.36 \mathrm{~cm}^{-1}$, smaller than that of $\nu_{7}$. For this reason, the higher-to-higher band is actually lower than the lower-to-lower band, provided that the NN-PES is accurate. This may be the reason why Dong et al. did not observe the higher-to-higher band. It could also be that because the initial state is $13.90 \mathrm{~cm}^{-1}$ higher, its population is low enough that the transition is not observed. Dong et al. comment that it is surprising that vibrational excited hydrocarbon radicals survived in their slit jet experiment. 


\section{CONCLUSION}

The vinyl radical (VR) is an important test molecule for variational methods. Variational calculations are difficult when simple basis size reduction and potential representation ideas fail [66], as they do for VR. Because VR has five atoms and two equivalent equilibrium structures, calculations with a direct product basis, though possible for low-lying levels, are challenging. It is common to prune a direct product basis by discarding some of its functions. [67-74] The most popular pruning schemes work best for single-well molecules. Potential integrals are another problem. Because VR has five atoms, one must either re-represent the PES in a special form designed to facilitate the evaluation of potential integrals or deal with 9D quadrature. There are methods that do not require storing vectors with as many elements as there are basis functions, for which basis size reduction is not necessary, [75-77] but they are also only applicable if the PES has a special form. Many variational methods are designed to be used with normal coordinates and they would work poorly for VR. The variational method we use in this paper uses contracted basis functions, polyspherical coordinates, and the Lanczos algorithm. The basis functions are products of bend and stretch functions, $|b s\rangle$. For VR, it is possible to use enough contracted basis functions to converge many energy levels. This requires solving the final eigenvalue problem with an iterative method, in our case the Lanczos algorithm. A key challenge one must confront when doing this is the memory cost of storing a matrix representing $\Delta V$ in the $|b s\rangle$ basis. We store an intermediate matrix called the $\mathbf{F}$ matrix and evaluate matrix-vector products by doing sums sequentially.

Owing to the fact that is an interesting challenge, two other groups have previously done variational calculations for VR.[3,4] Their results do not agree and differ also from those of this paper. One source of discrepancy is the PES. Variational calculations for VR are only possible because there is a PES.[1] The authors of Ref. 1 assumed that their PES had no symmetry errors because of the exchange of Cartesian coordinates they built into their PES routine. However, at many points with $R_{\mathrm{C}_{5}-\mathrm{H}_{2}}=R_{\mathrm{C}_{5}-\mathrm{H}_{3}}$, the PES of Ref. 1 does have symmetry errors. S̆mydke et al. recognized that the PES of Ref. 1 was not invariant wrt (23) and corrected this error by averaging. Unfortunately, to use the PES routine of Ref. 1 with a KEO and basis functions in internal coordinates, it is necessary to transform from internal coordinates to Cartesian coordinates and due to round-off error, at a subset of the 
points with $R_{\mathrm{C}_{5}-\mathrm{H}_{2}}=R_{\mathrm{C}_{5}-\mathrm{H}_{3}}$, the potential is therefore not invariant wrt $E^{*}$. The PES of Ref. 4 inherits this problem. In this paper we identify and correct the $E^{*}$ problem. At lower energies, where it seems that the (23) and $E^{*}$ errors are less important, levels computed by $\mathrm{Yu}$ et al. are close to our energies. It is almost certain that it is only the symmetry errors that are responsible for differences between our levels and those of Yu et al. We do not know what causes differences between our levels and those of Šmydke et al.

For VR, we calculate tunnelling splittings. Some of tunnelling splittings from the three calculations are very close but others even have different signs. There are accurate experimental tunnelling splittings for the ground state, $\nu_{3}$, and $\nu_{8}$, from high-resolution spectroscopic studies. Except for $\nu_{3}$, the theoretical tunnelling splittings are in good agreement with experimental values. The theoretical and experimental frequencies for $\nu_{3}$ and $\nu_{8}$ are also in good agreement. For some states, the most important tunnelling path is the one that corresponds to the bending of the $\mathrm{H}$ atom of the $\mathrm{CH}$ group. However, for others, an out of plane tunnelling path is significant. We are able to compute and assign enough levels that we can determine hot band transition frequencies. The hot band at $2897.23 \mathrm{~cm}^{-1}$, corresponding to the lower-to-lower transition of the $\nu_{7} \rightarrow \nu_{3}+\nu_{7}$, observed by F. Dong et al. [10] is in good agreement with our calculated value of $2895.90 \mathrm{~cm}^{-1}$. The $4.63 \mathrm{~cm}^{-1}$ experimental red-shift of this hot band is in excellent agreement with the calculated value of $4.47 \mathrm{~cm}^{-1}$. There is therefore considerable evidence indicating that the NN-PES is quite accurate. However, we know that it must be possible to improve it by fixing the original (23) symmetry error. Rather than exchanging Cartesian coordinates as in Ref. 1 and ad hoc averaging as in Ref. 4, it would be better to fit the ab initio points with a permutation invariant neuron network[78, 79].

\section{SUPPLEMENTARY MATERIAL}

A potential contour plot to reveal the hole on the original NN-PES. 


\section{Appendix A: Symmetry errors of the $\mathrm{C}_{2} \mathrm{H}_{3}$ PESs}

In this paper we refer to three $\mathrm{C}_{2} \mathrm{H}_{3}$ PESs. All the PESs are functions of Cartesian coordinates. The first PES is the original PES of Ref. 1. We denote it $V_{\mathrm{NN}}$. The authors of Ref. 1 attempt to make their PES invariant with respect to (wrt) (23), the permutation of hydrogen atoms 2 and 3. They do this by inserting into their code statements before calling the NN expansion functions:

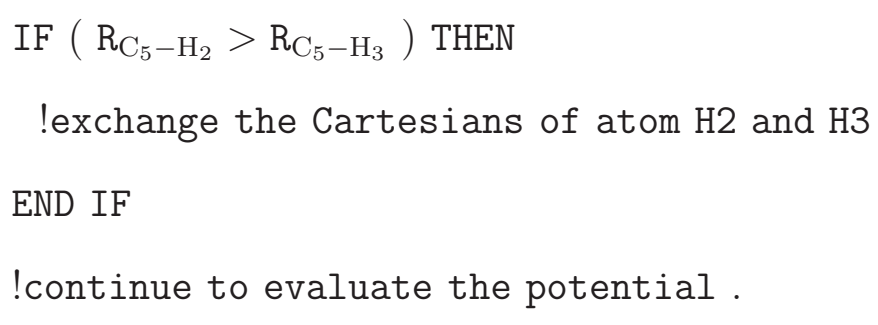

Unfortunately, $V_{\mathrm{NN}}$ is not invariant wrt (23) when $R_{\mathrm{C}_{5}-\mathrm{H}_{2}}=R_{\mathrm{C}_{5}-\mathrm{H}_{3}}$. Yu uses $V_{\mathrm{NN}}$. [3] The second PES is the PES of Ref. 4, denoted by $V_{\text {sym }}$,

$$
V_{\mathrm{sym}}\left(\mathrm{H}_{2}, \mathrm{H}_{3}\right)=\frac{1}{2}\left[V_{\mathrm{NN}}\left(\mathrm{H}_{2}, \mathrm{H}_{3}\right)+\mathrm{V}_{\mathrm{NN}}\left(\mathrm{H}_{3}, \mathrm{H}_{2}\right)\right] .
$$

They fix the permutation symmetry error by averaging. The averaging successfully corrects the permutation error of $V_{\mathrm{NN}}$. Unfortunately, $V_{\mathrm{sym}}(A) \neq V_{\mathrm{sym}}\left(A^{*}\right)$ for some $A$ having $R_{\mathrm{C}_{5}-\mathrm{H}_{2}}=R_{\mathrm{C}_{5}-\mathrm{H}_{3}}$, where $A$ and $A^{*}$ represent sets of Radau-Jacobi coordinates (or other internal coordinates). $A^{*}$ is the set of Radau-Jacobi coordinates obtained by applying the inversion operator $E^{*}$ to the $A$ Radau-Jacobi coordinates. $V_{\text {sym }}(A)$ means call the PES routine at Cartesian coordinates computed from the $A$ Radau-Jacobi coordinates. In this appendix, we first evaluate the importance of the (23) symmetry error of $V_{\mathrm{NN}}$ and then present a recipe for creating a PES that is invariant wrt to both (23) and $E^{*}$.

$V_{\mathrm{NN}}$, used by $\mathrm{Yu}$, has symmetry errors, when $R_{\mathrm{C}_{5}-\mathrm{H}_{2}}=R_{\mathrm{C}_{5}-\mathrm{H}_{3}}$. They have a significant effect on the reference bend potentials they and we use to solve for the bend eigenfunctions, because they are evaluated at $R_{\mathrm{C}_{5}-\mathrm{H}_{2}}=R_{\mathrm{C}_{5}-\mathrm{H}_{3}}$. Here we define error as the difference between $V_{\mathrm{NN}}(A)$ and $V_{\mathrm{NN}}((23) A)$. Among the 5 -D bend quadrature points under the ceiling value given in Table II, the largest error is $1908 \mathrm{~cm}^{-1}$ and occurs for a pair of points with potential values 20110 and $22018 \mathrm{~cm}^{-1}$. Points above the ceiling have even larger errors. For lower energies, the errors are smaller, but still significant, e.g., an error of $18 \mathrm{~cm}^{-1}$ at a point 
with potential $52 \mathrm{~cm}^{-1}$. This low-energy point is on a path that we shall now examine. It is close to the minimum energy path for the tunnelling of $\mathrm{H}_{1}$ and obtained by scanning $\theta_{1}$ and fixing all of the other polyspherical coordinates associated with the bond vectors equal to their values at the saddle point. See Fig. 1 for an illustration of the coordinates. We know that the path is close to the minimum energy path because at the lowest point on the path $\left(\theta_{1}=41.8^{\circ}\right)$, the energy is only $51.68 \mathrm{~cm}^{-1}$ higher than the global minima. At the highest point on the path $\left(\theta_{1}=0\right)$, the energy is $1743.13 \mathrm{~cm}^{-1}$, the saddle point energy. We compare $V_{\mathrm{NN}}(A)$, where $A$ is a point on the path, and $V_{\mathrm{NN}}((23) A)$; the two should be equal.

$$
V_{\mathrm{NN}}(A) \quad \text { is } \quad V_{\mathrm{NN}}\left(r_{0}, r_{1}, r_{2}, r_{3} ; \theta_{1}, \theta_{2}, \theta_{3}, \phi_{2}, \phi_{3}\right) \quad \text { and } \quad V_{\mathrm{NN}}((23) A) \quad \text { is }
$$

$V_{\mathrm{NN}}\left(r_{0}, r_{1}, r_{3}, r_{2} ; \theta_{1}, \theta_{3}, \theta_{2}, \phi_{3}, \phi_{2}\right)$. In Fig. 7 , we plot the minimum (blue) and maximum (red) of the two values. If there were no symmetry errors, the red and blue curves would be on top of each other. We plot only half of the double well because the other half is the same. See Fig. 7. The PES code takes Cartesian coordinates as input, which we compute from the polyspherical coordinates, and computes the distances $R_{\mathrm{C}_{5}-\mathrm{H}_{2}}$ and $R_{\mathrm{C}_{5}-\mathrm{H}_{3}}$ used to do the test of Eq. (A1). The symmetry errors along the path of Fig. 7, are largest close to the minimum. This symmetry error is visible in the inset of Fig. 7. Averaging using Eq. (A2) removes the (23) symmetry error at all the 5-D quadrature points.

Unfortunately, $V_{\text {sym }}$, which completely solves the symmetry problem of the previous paragraph, is, in practice, not invariant wrt $E^{*}$ everywhere. $V_{\text {sym }}(A)=V_{\text {sym }}\left(A^{*}\right)$, if and only if the average of $V_{\mathrm{NN}}(A)$ and $V_{\mathrm{NN}}((23) A)$ and the average of $V_{\mathrm{NN}}\left(A^{*}\right)$ and $V_{\mathrm{NN}}\left((23) A^{*}\right)$ are equal. When used to calculate energy levels, this condition is not always satisfied because for some $A$ with $R_{\mathrm{C}_{5}-\mathrm{H}_{2}}=R_{\mathrm{C}_{5}-\mathrm{H}_{3}} V_{\mathrm{NN}}(A) \neq V_{\mathrm{NN}}\left(A^{*}\right)$. What causes the inequality? The potential routine takes Cartesian coordinates as input. The problem is caused by the fact that the internal coordinates for which the potential routine is called are not exactly consistent with the bond distances inside the potential routine which are used to determine whether $R_{\mathrm{C}_{5}-\mathrm{H}_{2}}>R_{\mathrm{C}_{5}-\mathrm{H}_{3}}$ and whether to permute. Even if the bond lengths that correspond to a point satisfy $R_{\mathrm{C}_{5}-\mathrm{H}_{2}}=R_{\mathrm{C}_{5}-\mathrm{H}_{3}}$ outside the potential routine, it may be true that $R_{\mathrm{C}_{5}-\mathrm{H}_{2}} \neq R_{\mathrm{C}_{5}-\mathrm{H}_{3}}$ inside the potential routine, due to the round-off error of sine and cosine functions used to calculate the Cartesian coordinates of $A$ and $A^{*}$. The round-off error is a few $10^{-16}$. This tiny change is significant enough to trigger the IF test of Eq. (A1) for point $\mathrm{A}^{*}$, and not to trigger the same test for point A when the round-off error does not appear. 
Note that if energy levels were computed directly in Cartesian coordinates, so that conversion from internal to Cartesians was not required, then there would be no parity problem. Round-off error may make $V_{\mathrm{NN}}(A) \neq V_{\mathrm{NN}}\left(A^{*}\right)$ and hence $V_{\mathrm{sym}}(A) \neq V_{\mathrm{sym}}\left(A^{*}\right)$. To remove the $E^{*}$ symmetry error, we modify $V_{\mathrm{NN}}$ by replacing in the code of Ref. 1 their IF test with

$$
\operatorname{IF}\left(\left(\mathrm{R}_{\mathrm{C}_{5}-\mathrm{H}_{2}}-\mathrm{R}_{\mathrm{C}_{5}-\mathrm{H}_{3}}\right)>6 \mathrm{~d} 0 * \operatorname{epsilon}\left(\mathrm{R}_{\mathrm{C}_{5}-\mathrm{H}_{2}}\right)\right) \text { THEN }
$$

where the Fortran function epsilon() returns $2.22 \times 10^{-16}$ for a double precision real argument. We call the modified potential $\tilde{V}_{\mathrm{NN}}$. The potential we use in our calculations is obtained from Eq. (A2) by replacing $V_{\mathrm{NN}}$ with $\tilde{V}_{\mathrm{NN}}$, and is denoted by $\tilde{V}_{\mathrm{sym}}$. Among the 5-D bend quadrature points under the ceiling value given in Table II, the largest symmetry error on $V_{\text {sym }}$ is $1757 \mathrm{~cm}^{-1}$ and occurs for a pair of points with potential values 20211 and 21969 $\mathrm{cm}^{-1}$.

Finally, we report the number of points affected by symmetry errors on $V_{\mathrm{NN}}$ and on $V_{\text {sym. }}$. For the 5 -D bend quadrature points under the ceiling given in Table II, $66 \%$ of points on $V_{\mathrm{NN}}$ and $21 \%$ of points on $V_{\mathrm{sym}}$ are affected by the symmetry error. However, in the 9 -D calculation, for the stretch basis given in Table II, about $10 \%$ of points have $R_{\mathrm{C}_{5}-\mathrm{H}_{2}}=R_{\mathrm{C}_{5}-\mathrm{H}_{3}}$. Therefore the percentage of points affected by both symmetry errors is smaller in the 9-D calculation. This is probably the reason that why the low-lying levels computed on $V_{\mathrm{NN}}$ are not much affected by the symmetry error.

\section{Appendix B: Data Availability Statement}

The data that support the findings of this study are available from the corresponding author upon reasonable request.

\section{Acknowledgments}

This work has been supported by the Natural Sciences and Engineering Research Council of Canada. Calculations were done on the Compute Canada computer Beluga. We thank Jan S̆mydke and Attila G. Császá for sending us a copy of the potential surface of Ref. 1. 
We thank Hua-Gen Yu, Jan S̆mydke, and Attila G. Császá for helpful discussions.

[1] L. Chen, K. Shao, J. Chen, M. Yang and D. H. Zhang, J Chem. Phys. 144, 194309 (2016).

[2] A. R. Sharma, B. J. Braams, S. Carter, B. C. Shepler and J. M. Bowman, J Chem. Phys. 130, 174301 (2009).

[3] H.-G. Yu, H. Song and M. Yang, J Chem. Phys. 146, 224307 (2017).

[4] J. S̆mydke, C. Fábri, J. Sarka, and A. G. Császár, Phys. Chem. Chem. Phys. 21, 3453 (2019).

[5] D. J. Nesbitt and F. Dong, Phys. Chem. Chem. Phys., 10, 2113. (2008)

[6] A. R. Sharma, J. M. Bowman and D. J. Nesbitt, J Chem. Phys. 136, 034305 (2012).

[7] V. Barone, J. Bloino, and M. Biczysko, Phys. Chem. Chem. Phys. 12, 1092-1101 (2010).

[8] H. Kanamori, Y. Endo, and E. Hirota, J Chem. Phys. 92, 197-205 (1990).

[9] K. Tanaka, M. Toshimitsu, K. Harada, and T. Tanaka, J Chem. Phys. 120, 3604-3618 (2004).

[10] F. Dong, M. Roberts and D. J. Nesbitt, J Chem. Phys. 128, 044305 (2008).

[11] M. B. Pushkarsky, A. M. Mann, J. S. Yeston, and C. B. Moore, J. Chem. Phys. 115, 10738 (2001).

[12] P. L. Raston, T. Liang, and G. E. Douberly, J Chem. Phys. 138, 174302 (2013).

[13] Y. J. Wu, M. Y. Lin, B. M. Cheng, H. F. Chen, and Y. P. Lee,

J. Chem. Phys. 128, 204509 (2008).

[14] H. Tanskanen, L. Khriachtchev, M. Rasanen, V. I. Feldman, F. F. Sukhov, A. Y. Orlov, and D. A. Tyurin, J. Chem. Phys. 123, 064318 (2005).

[15] L. Letendre, D. K. Liu, C. D. Pibel, J. B. Halpern, and H. L. Dai, J. Chem. Phys. 112, 9209 (2000).

[16] M. Nikow, M. J. Wilhelm, and H. L. Dai, J. Phys. Chem. A 113, 8857 (2009).

[17] M. J. Bramley and T. Carrington, Jr., J. Chem. Phys. 99, 8519 (1993)

[18] H.-G. Yu and J. Muckerman, J. Mol. Spectros., 214, 11-20 (2002)

[19] E. Matyus, G. Czako and A. G. Császár, J. Chem. Phys. 130, 134112 (2009);

[20] T. Carrington Adv. Chem. Phys. 163, 217-243 (2018)

[21] T. Carrington, J. Chem. Phys. 146, 120902 (2017)

[22] C. Fábri, M. Quack, and A. G. Császár The Journal of Chemical Physics 147, 134101 (2017) 
[23] Z. Bacic and J. C. Light, Annu. Rev. Phys. Chem. 40, 469 (1989)

[24] S. Carter and N. C. Handy, Comput. Phys. Rep. 5, 115 (1986)

[25] J. Tennyson, Comput. Phys. Rep. 4, 1 (1986)

[26] D. Luckhaus, J. Chem. Phys. 113, 1329 (2000)

[27] J. R. Henderson and J. Tennyson, Chem. Phys. Lett. 173, 133 (1990)

[28] J. C. Light and T. Carrington, Jr., Adv. Chem. Phys. 114, 263 (2000)

[29] J. M. Bowman and B. Gazdy, J. Chem. Phys. 94, 454 (1991)

[30] P. M. Felker, and Z. Bacic, J. Chem. Phys. 151, 024305 (2019)

[31] P. M. Felker, and Z. Bacic, J. Chem. Phys. 152, 014108 (2020); https://doi.org/10.1063/1.5138992

[32] M.J. Bramley and T. Carrington Jr. J. Chem. Phys. 101, 8494-8507 (1994)

[33] X.-G. Wang and T. Carrington Jr. J. Chem. Phys. 117, 6923 (2002)

[34] H.-G. Yu, J. Chem. Phys. 117, 8190 (2002).

[35] H.-G. Yu, J. Mol. Spectrosc. 256, 287 (2009).

[36] H.-G. Yu, J. Chem. Phys. 145, 084109 (2016).

[37] F. Gatti and C. Iung, Physics Reports, 484, 1-69 (2009)

[38] F. Smith, Phys. Rev. Lett. 45, 1157 (1980)

[39] X.-G. Wang and T. Carrington, Jr, J. Chem. Phys. 119, 101 (2003).

[40] D Xu, R Chen, H Guo The Journal of chemical physics 118, 7273-7282 (2003)

[41] W. T. S. Cole, R. S. Fellers, M. R. Viant, C. Leforestier, and R. J. Saykally, J. Chem. Phys. 143, 154306 (2015).

[42] J. Bowman, T. Carrington Jr, and H.-D. Meyer, Mol. Phys. 106, 2145-2182 (2008)

[43] M. Mladenović, J. Chem. Phys. 112, 1070 (2000).

[44] X.-G. Wang and T. Carrington, Jr, J. Chem. Phys. 118, 6946 (2003).

[45] X.-G. Wang and T. Carrington Jr, The Journal of Chemical Physics 141, 154106 (2014);

[46] J. Cullum and R. A. Willoughby, Lanczos Algorithm for Large Symmetric Eigenvalue Computations (Birkhäuser: Boston 1985).

[47] C.C. Paige, J. Inst. Math. Appl., 10373 (1972).

[48] P. R. Bunker and P. Jensen, Molecular Symmetry and Spectroscopy (NRC Research Press: Ottawa 1998). 
[49] X.-G. Wang and T. Carrington, Jr., J. Chem. Phys. 114, 1473 (2001).

[50] R. Chen and H. Guo, J. Chem. Phys. 114, 1467 (2001).

[51] X.-G. Wang and T. Carrington J. Chem. Phys. 129, 234102 (2008)

[52] H.-S. Lee and J.C. Light, J. Chem. Phys. 120, 4626, (2004).

[53] X.-G. Wang and T. Carrington, Jr., J. Chem. Phys. 123, 154303 (2005)

[54] X.-G. Wang, T. Carrington Jr, R. Dawes, A. W. Jasper Journal of Molecular Spectroscopy 268 53-65 (2011)

[55] J. Smydke and A. G. Császár, Molecular Physics, 117, 1682 (2019)

[56] G. Donoghue, X.-G. Wang, R. Dawes, and T. Carrington Jr. J. Mol. Spectrosc.hys. 330, 170 (2016)

[57] E. Castro-Juarez, X.-G. Wang, T. Carrington, E. Quintas-Sánchez, and R. Dawes J. Chem. Phys. 151, 084307 (2019)

[58] X.-G. Wang and T. Carrington, Jr, J. Chem. Phys. 121, 2937 (2004).

[59] H.-G. Yu, private communication, November, 2019

[60] J. S̆mydke, and A. G. Császár, private communication, Janunary, 2020

[61] M. J. Bramley, J. W. Tromp, T. Carrington, Jr., and G. C. Corey J. Chem. Phys. 1006175 (1994).

[62] C. Leforestier, L.B. Braly, K. Liu, M.J. Elrod, R.J. Saykally, J. Chem. Phys. 1068527 (1997).

[63] X.-G. Wang and T. Carrington, Jr., Comp. Phys. Comm. 181, 455 (2010).

[64] X.-G. Wang and T. Carrington, Jr., J. Chem. Phys. 148, 074108 (2018).

[65] C. Fábri, R. Marquardt, A. G. Császár, and Martin Quack J. Chem. Phys. 150, 014102 (2019)

[66] T. Carrington, Jr., J. Chem. Phys. 146, 120902 (2017)

[67] J.M. Bowman, S. Carter, X. Huang International Reviews in Physical Chemistry 22, 533-549 (2003).

[68] L. Halonen, D. W. Noid, and M. S. Child, J. Chem. Phys. 78, 2803 (1983).

[69] B. Poirier, J. Theor. Comput. Chem. 2, 65 (2003)

[70] Marat Sibaev and Deborah L. Crittenden J. Chem. Phys. 145, 064106 (2016)

[71] G. Rauhut, J. Chem. Phys. 127, 184109 (2007)

[72] X.-G. Wang and T. Carrington, Jr., J. Phys. Chem. A 105, 2575 (2001)

[73] G Avila, T Carrington Jr The Journal of chemical physics 137 (17), 174108 
[74] R Wodraszka and T.Carrington J. Chem. Phys 145, 04411018 (2016)

[75] A. Leclerc and T. Carrington J. Chem. Phys. 140, 174111 -1 - 174111-13 (2014)

[76] Maxim Rakhuba and Ivan Oseledets The Journal of Chemical Physics 145, 124101 (2016)

[77] H. R. Larsson The Journal of Chemical Physics 151 (20), 204102 (2019)

[78] B. Jiang and H. Guo, J. Chem. Phys. 139, 054112 (2013).

[79] K. Shao, J. Chen, Z. Zhao, and D. H. Zhang J. Chem. Phys. 145, 071101 (2016). 


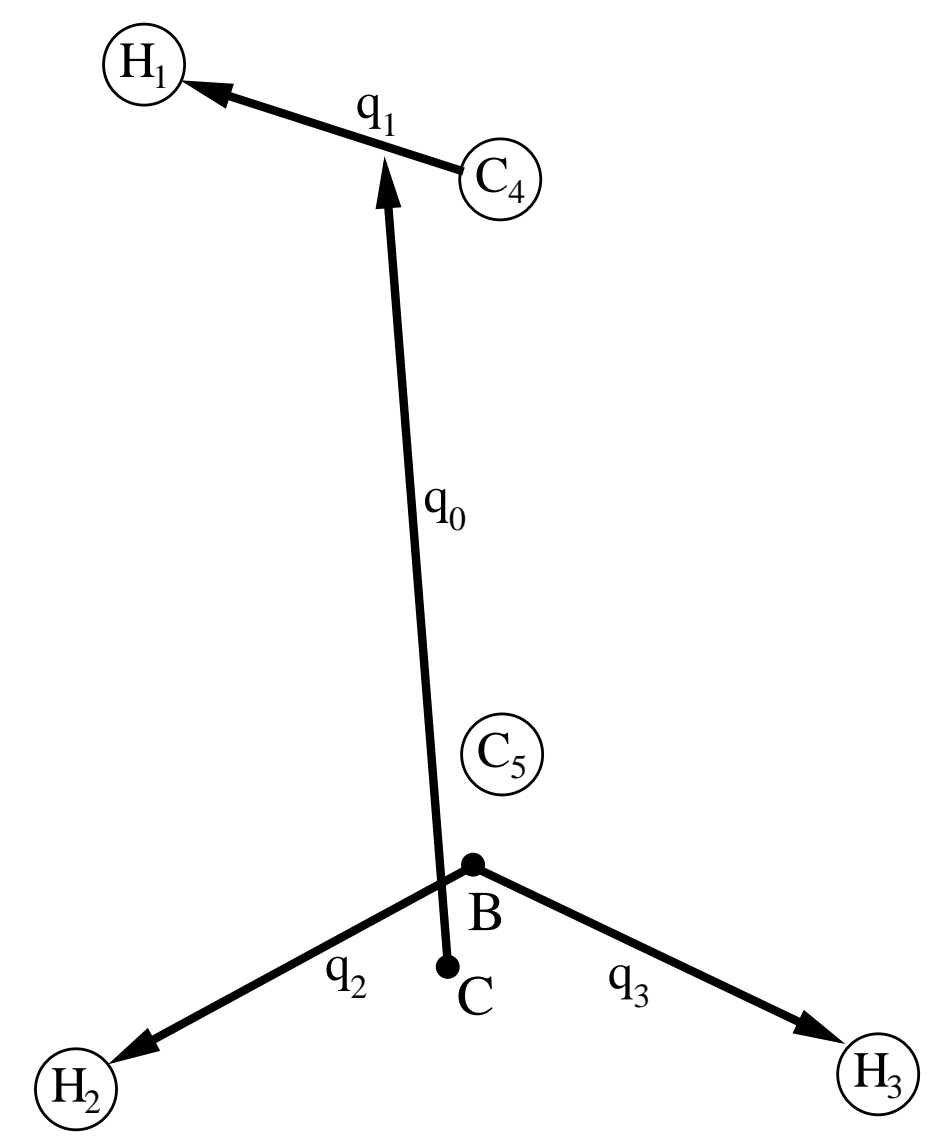

FIG. 1: Radau-Jacobi vectors for $\mathrm{CH}_{2}-\mathrm{CH}$. qo is the Jacobi vector from the center of mass of $\mathrm{C}_{5^{-}}$ $\mathrm{H}_{2}-\mathrm{H}_{3}$ to the center of mass of $\mathrm{C}_{4}-\mathrm{H}_{1} \cdot \mathbf{q}_{2}$ and $\mathbf{q}_{3}$ are $1+2$ Radau vectors for the $\mathrm{H}_{2}-\mathrm{C}_{5}-\mathrm{H}_{3}$ group. $\mathrm{B}$ and $\mathrm{C}$ are the Radau canonical point and the center of mass of $\mathrm{CH}_{2}$, respectively. We use the polyspherical angles $\left(\theta_{1}, \theta_{2}, \theta_{3}, \phi_{2}, \phi_{3}\right)$ associated with these four vectors to compute levels. Bond vectors $\left(\mathbf{b}_{\mathbf{0}}, \mathbf{b}_{\mathbf{1}}, \mathbf{b}_{\mathbf{2}}, \mathbf{b}_{\mathbf{3}}\right)$ (not shown) are $\left(\overrightarrow{\mathrm{C}_{5} \mathrm{C}_{4}}, \overrightarrow{\mathrm{C}_{4} \mathrm{H}_{1}}, \overrightarrow{\mathrm{C}_{5} \mathrm{H}_{2}}, \overrightarrow{\mathrm{C}_{5} \mathrm{H}_{3}}\right)$. Bond vectors are computed from the Radau-Jacobi vectors using a transformation matrix (given in the text) and are then used to compute Cartesian coordinates of each atom which are used to evaluate the potential. 
FIG. 2: Wavefunction cuts for $\nu_{5}(A+)$ and $2 \nu_{5}(A+)$, computed from 5-D bend wavefunctions by fixing the other three Radau-Jacobi angles at their saddle point values. The contour interval is 0.25. The same parameters are adopted for the wavefunction cuts in Figs. 3, 4 and 5 . 

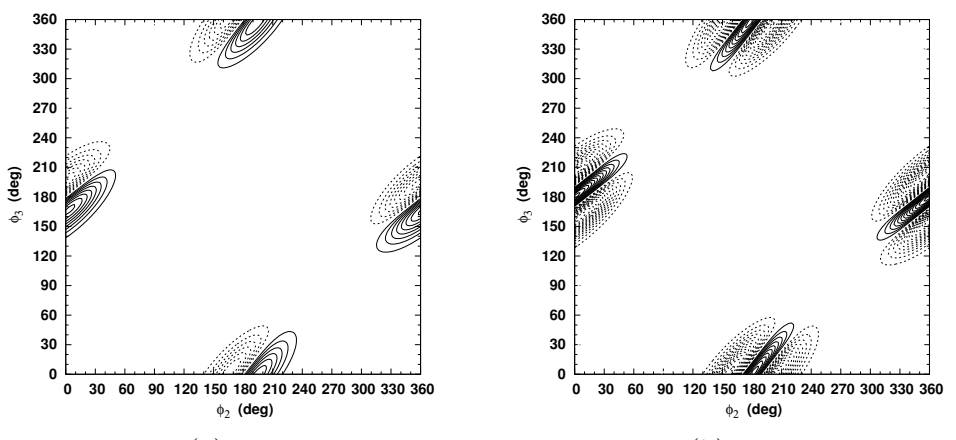

(a) $\nu_{8}$

(b) $2 \nu_{8}$

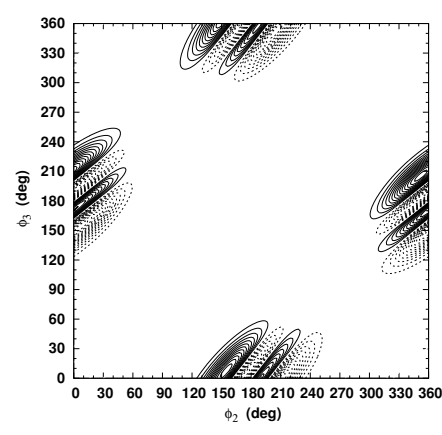

(c) $3 \nu_{8}$

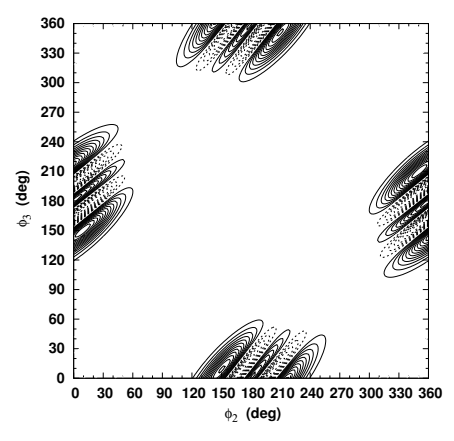

(d) $4 \nu_{8}$

FIG. 3: Wavefunction cuts for $\nu_{8}(A-), 2 \nu_{8}(A+), 3 \nu_{8}(A-)$, and $4 \nu_{8}(A+)$ obtained from 5 -D bend wavefunctions. 

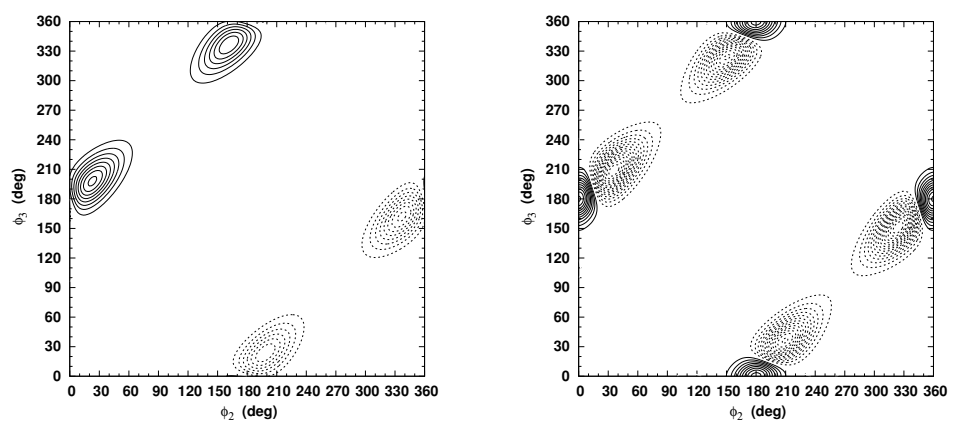

(a) $\nu_{9}$

(b) $2 \nu_{9}$

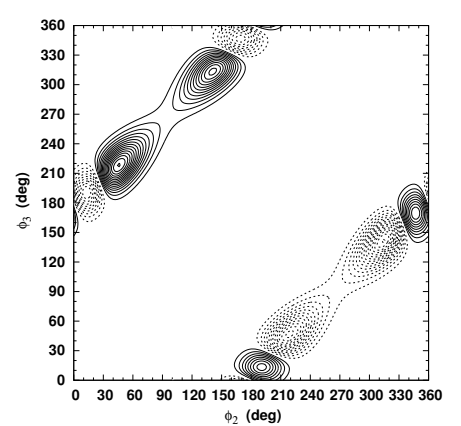

(c) $3 \nu 9$

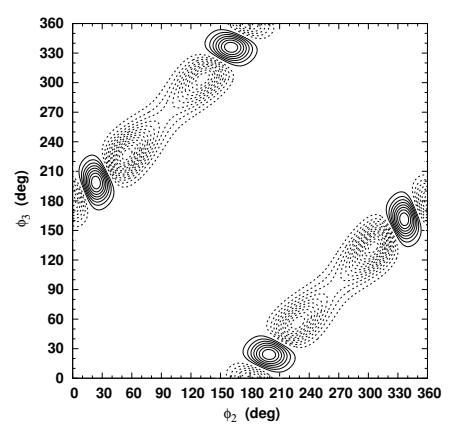

(d) $4 \nu 9$

FIG. 4: Wavefunction cuts for $\nu_{9}(A-), 2 \nu_{9}(A+), 3 \nu_{9}(A-)$, and $4 \nu_{9}(A+)$, obtained from 5 -D bend wavefunctions.

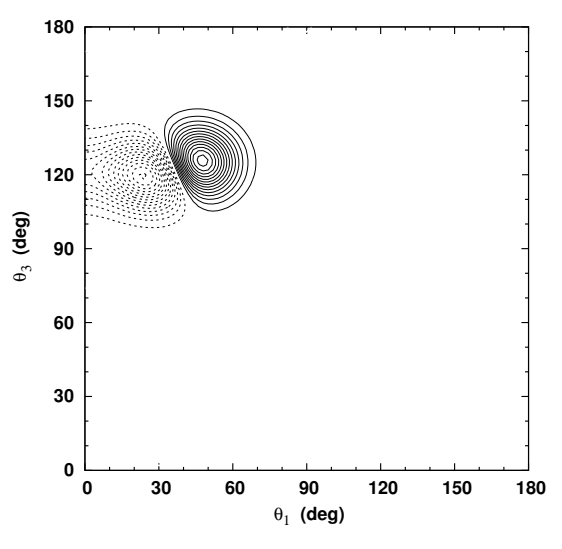

(a) $\nu_{7}$

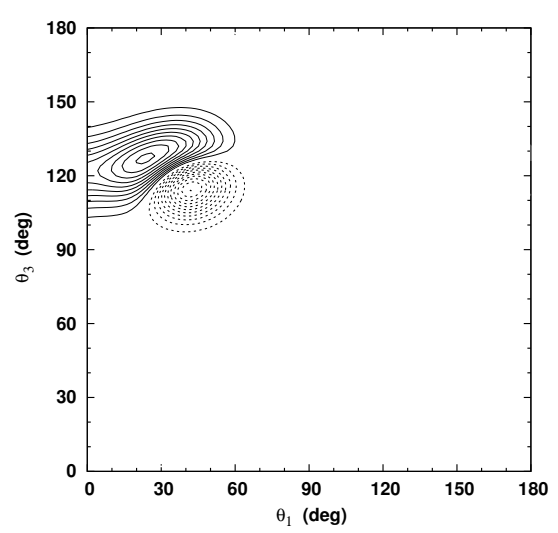

(b) $\nu_{6}$

FIG. 5: Wavefunction cuts for $\nu_{6}(A+)$ and $\nu_{7}(A+)$, obtained from 5-D bend wavefunctions. 

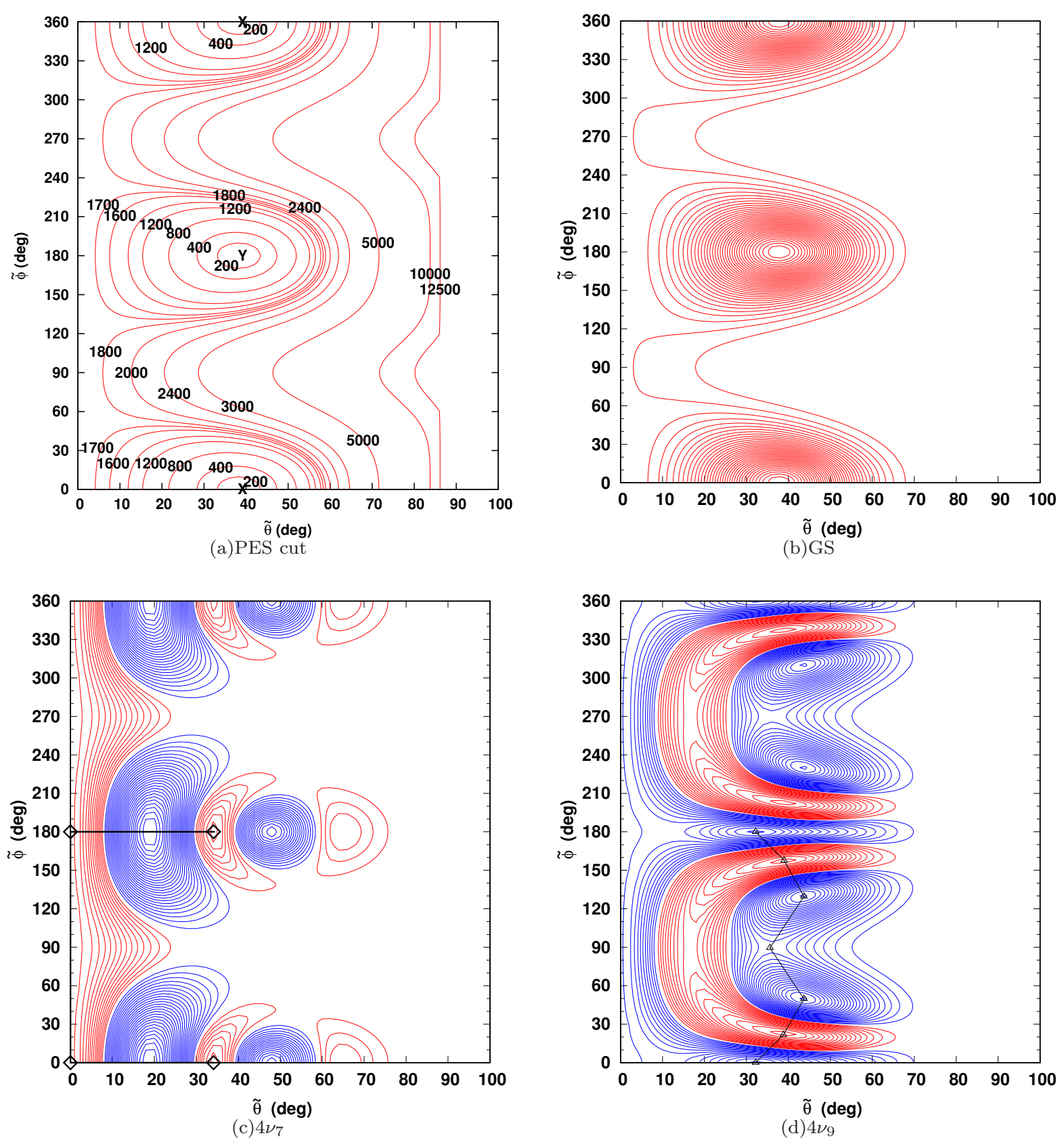

FIG. 6: (a) 2-D $(\tilde{\theta}, \tilde{\phi})$ PES cut of 5-D $V_{\text {bref }}$ computed by fixing the other three Radau-Jacobi angles at their saddle point values. The two equivalent global minima are marked as $\mathbf{X}$ and $\mathbf{Y}$. (b) Wavefunction cut for the ground state $(A+)$. (c) Wavefunction cut for $4 \nu_{7}(A+)$. For this state, the $\mathrm{C}_{\alpha}-\mathrm{H}$ tunneling occurs along the in-plane path indicated by the black line with four diamonds. The path goes through the saddle point at the $\tilde{\theta}=0$ vertical line. (d) Wavefunction cut for $4 \nu_{9}(A+)$. For this state, the $\mathrm{C}_{\alpha}-\mathrm{H}$ tunneling occurs via a secondary out-of-plane path indicated by the black lines linking the wavefunction peaks. Red and blue lines are positive and negative values, respectively. The contour interval is 0.25 . 


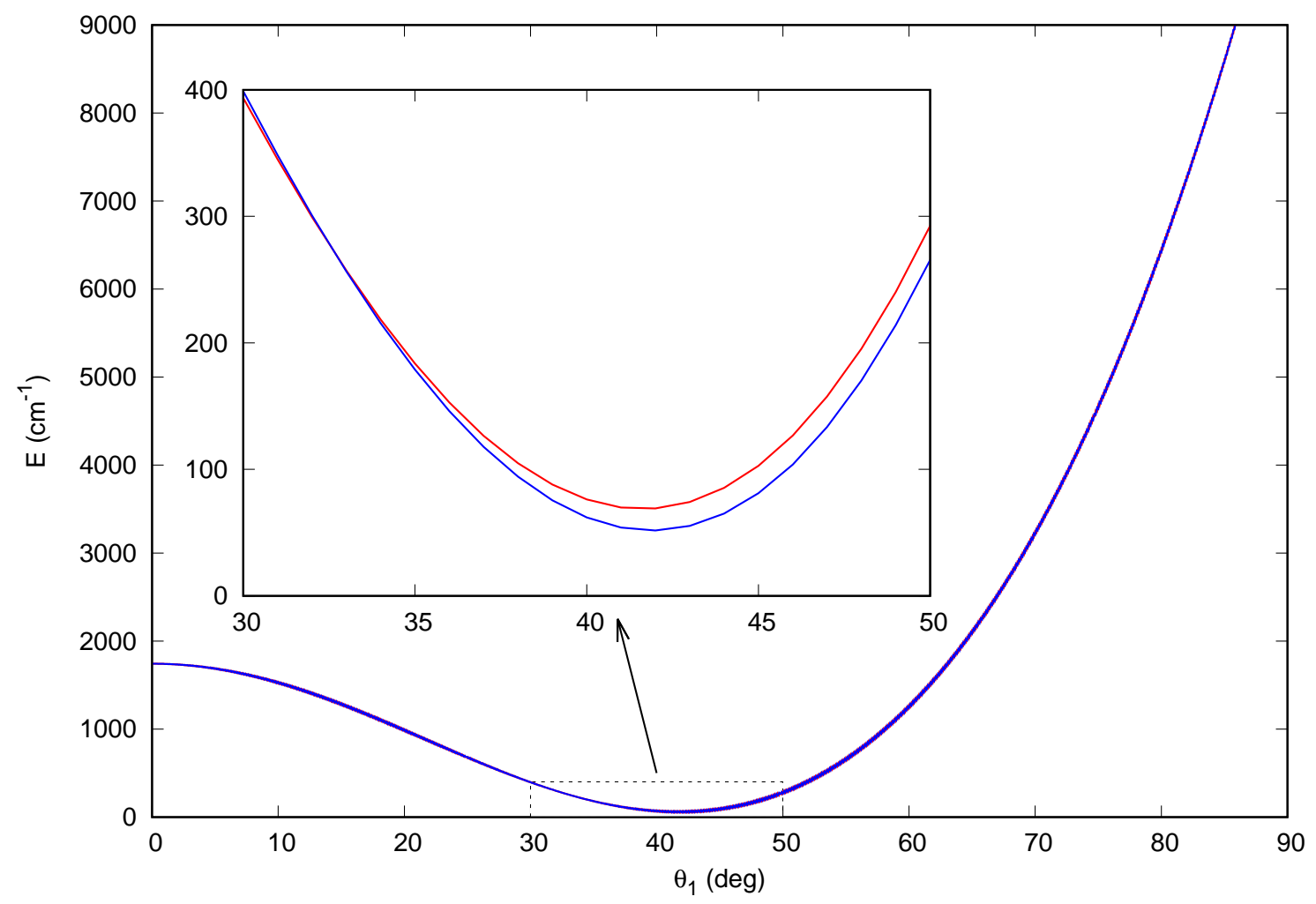

FIG. 7: A $\theta_{1}$ 1-D potential cut in bond coordinates obtained by fixing all the other coordinates equal to their saddle point values. For each point on this curve, we evaluate $V_{\mathrm{NN}}\left(\mathrm{H}_{2}, \mathrm{H}_{3}\right)$ and $V_{\mathrm{NN}}\left(\mathrm{H}_{3}, \mathrm{H}_{2}\right)$ and plot the minimum (blue) and maximum (red) of the two values. The difference bewteen the red and blue curves (only visible in the inset) is due to the (23) symmetry error of NN-PES. See the Appendix for details. 
TABLE I: Stationary points of $\mathrm{CH}_{2}-\mathrm{CH}$ on the NN-PES[1]. Distances are in bohr and angles are in degrees. Bond vectors $\left(\mathbf{b}_{\mathbf{0}}, \mathbf{b}_{\mathbf{1}}, \mathbf{b}_{\mathbf{2}}, \mathbf{b}_{\mathbf{3}}\right)$ are $\left(\overrightarrow{\mathrm{C}_{5} \mathrm{C}_{4}}, \overrightarrow{\mathrm{C}_{4} \mathrm{H}_{1}}, \overrightarrow{\mathrm{C}_{5} \mathrm{H}_{2}}, \overrightarrow{\mathrm{C}_{5} \mathrm{H}_{3}}\right)$. The bond vector lengths are $\left(R_{\mathrm{C}_{5}-\mathrm{C}_{4}}, R_{\mathrm{C}_{4}-\mathrm{H}_{1}}, R_{\mathrm{C}_{5}-\mathrm{H}_{2}}, R_{\mathrm{C}_{5}-\mathrm{H}_{3}}\right)$.

\begin{tabular}{lcc}
\hline \hline & Minimum & Saddle point \\
\hline Bond vectors & & \\
$\mathrm{R}_{\mathrm{C}_{5}-\mathrm{C}_{4}}$ & 1.31233 & 1.30366 \\
$\mathrm{R}_{\mathrm{C}_{4}-\mathrm{H}_{1}}$ & 1.07794 & 1.06451 \\
$\mathrm{R}_{\mathrm{C}_{5}}-\mathrm{H}_{2}$ & 1.09038 & 1.09078 \\
$\mathrm{R}_{\mathrm{C}_{5}-\mathrm{H}_{3}}$ & 1.08488 & 1.09078 \\
$\theta_{\mathrm{H}_{1}-\mathrm{C}_{4}-\mathrm{C}_{5}}$ & 137.45 & 115.89 \\
$\theta_{\mathrm{H}_{4}-\mathrm{C}_{5}-\mathrm{H}_{2}}$ & 121.36 & 115.89 \\
$\theta_{\mathrm{H}_{4}-\mathrm{C}_{5}-\mathrm{H}_{3}}$ & 122.02 & 115.89 \\
& & \\
$\mathrm{R}_{\text {adau-Jacobi vectors }}$ & & \\
$R_{0}$ & 1.45709 & 1.46980 \\
$R_{1}$ & 1.07994 & 1.06451 \\
$R_{2}$ & 1.06842 & 1.06823 \\
$R_{3}$ & 1.06323 & 1.06823 \\
$\theta_{1}$ & 40.36 & 0.00 \\
$\theta_{2}$ & 117.23 & 120.26 \\
$\theta_{3}$ & 122.25 & 120.26 \\
$\phi_{2}$ & 0.00 & 0.00 \\
$\phi_{3}$ & 180.00 & 180.00 \\
& & \\
$V\left(\mathrm{~cm}^{-1}\right)$ & 0.00 & 1743.130 \\
\hline \hline
\end{tabular}


TABLE III: Vibrational energy levels (in $\mathrm{cm}^{-1}$ ) of $\mathrm{C}_{2} \mathrm{H}_{3}$ with $A+\left(A_{1}\right)$ symmetry. Cal1 is this paper. Cal2 and Cal3 are levels from S̆mydke et al. [4] and Yu et al. [3], respectively. $\Delta_{12}=$ Cal1 - Cal2, $\Delta_{13}=$ Cal1 - Cal3. The numbers in parentheses in the Cal1 column are the leading coefficients of the wavefunctions. Assign1 and Assign2 are the assignments of Cal1 and Cal2, respectively. "yes" in Assign1 means that the assignment of Assign1 and Assign2 agree. "no" in Assign1 means that no assignment can be made. All the levels are in order of increasing energy. The zero point energy of Cal1 is $7870.04 \mathrm{~cm}^{-1}$.

\begin{tabular}{|c|c|c|c|c|c|c|c|}
\hline$i$ & Cal1 & Cal2 & Cal3 & $\Delta_{12}$ & $\Delta_{13}$ & Assign1 & Assign2 \\
\hline 1 & $0.00(0.98)$ & .0 & .0 & 0.0 & 0.0 & yes & GS \\
\hline 2 & $667.66(0.96)$ & 665.9 & 667.5 & 1.8 & 0.2 & yes & $\nu_{7}$ \\
\hline 3 & $996.44(0.97)$ & 991.4 & 996.3 & 5.0 & 0.1 & yes & $\nu_{6}$ \\
\hline 4 & $1247.71(0.95)$ & 1246.0 & 1247.5 & 1.7 & 0.2 & yes & $2 \nu_{7}$ \\
\hline 5 & $1358.13(0.86)$ & 1355.2 & 1357.8 & 2.9 & 0.3 & yes & $\nu_{5}$ \\
\hline 6 & $1501.75(0.98)$ & 1501.1 & 1500.9 & 0.7 & 0.9 & yes & $2 \nu_{9}$ \\
\hline 7 & $1569.51(0.96)$ & 1554.4 & 1569.2 & 15.1 & 0.3 & yes & $\nu_{6}+\nu_{7}$ \\
\hline 8 & $1583.88(0.84)$ & 1583.3 & 1583.6 & 0.6 & 0.3 & yes & $\nu_{4}$ \\
\hline 9 & $1647.83(0.97)$ & 1647.4 & 1646.8 & 0.4 & 1.0 & yes & $\nu_{8}+\nu_{9}$ \\
\hline 10 & $1781.57(0.97)$ & 1780.8 & 1781.2 & 0.8 & 0.4 & yes & $2 \nu_{8}$ \\
\hline 11 & $1851.64(0.95)$ & 1841.8 & 1851.3 & 9.8 & 0.3 & yes & $3 \nu_{7}$ \\
\hline 12 & $2012.27(0.97)$ & 1992.4 & 2012.4 & 19.9 & -0.1 & yes & $2 \nu_{6}$ \\
\hline 13 & $2026.76(0.85)$ & 2016.4 & 2027.0 & 10.4 & -0.2 & yes & $\nu_{5}+\nu_{7}$ \\
\hline 14 & $2127.28(0.96)$ & 2125.2 & 2127.1 & 2.1 & 0.2 & yes & $\nu_{7}+2 \nu_{9}$ \\
\hline 15 & $2220.49(0.92)$ & 2192.2 & 2220.3 & 28.3 & 0.2 & no & $\nu_{6}+2 \nu_{7}$ \\
\hline 16 & $2249.89(0.80)$ & 2244.7 & 2249.8 & 5.2 & 0.1 & yes & $\nu_{4}+\nu_{7}$ \\
\hline 17 & $2300.05(0.95)$ & 2297.5 & 2299.9 & 2.5 & 0.1 & yes & $\nu_{7}+\nu_{8}+\nu_{9}$ \\
\hline 18 & $2349.80(0.86)$ & 2327.7 & 2351.6 & 22.1 & -1.8 & yes & $\nu_{5}+\nu_{6}$ \\
\hline 19 & $2453.55(0.80)$ & 2447.9 & 2453.8 & 5.7 & -0.2 & yes & $\nu_{7}+2 \nu_{8}$ \\
\hline 20 & $2466.70(0.81)$ & 2459.8 & 2467.2 & 6.9 & -0.5 & yes & $\nu_{6}+2 \nu_{9}$ \\
\hline 21 & $2555.35(0.90)$ & 2511.7 & 2570.8 & 43.6 & -15.5 & yes & $4 \nu_{7}$ \\
\hline 22 & $2570.55(0.82)$ & 2563.9 & 2584.2 & 6.6 & -13.7 & yes & $\nu_{4}+\nu_{6}$ \\
\hline 23 & $2609.77(0.82)$ & 2599.4 & 2611.4 & 10.4 & -1.6 & no & $\nu_{5}+2 \nu_{7}$ \\
\hline 24 & $2633.48(0.96)$ & 2625.2 & 2634.1 & 8.3 & -0.6 & no & $\nu_{6}+\nu_{8}+\nu_{9}$ \\
\hline 25 & $2651.57(0.88)$ & 2634.8 & 2709.6 & 16.8 & -58.0 & yes & $2 \nu_{7}+2 \nu_{9}$ \\
\hline 26 & $2708.02(0.76)$ & 2695.4 & 2710.4 & 12.6 & -2.4 & $2 \nu_{5}$ & $2 \nu_{6}+\nu_{7}$ \\
\hline 27 & $2709.26(0.87)$ & 2696.9 & 2784.8 & 12.4 & -75.5 & no & $2 \nu_{5}$ \\
\hline 28 & $2783.62(0.96)$ & 2773.9 & 2828.5 & 9.7 & -44.9 & yes & $\nu_{6}+2 \nu_{8}$ \\
\hline 29 & $2828.16(0.77)$ & 2819.1 & 2855.7 & 9.1 & -27.5 & yes & $\nu_{4}+2 \nu_{7}$ \\
\hline 30 & $2854.94(0.91)$ & 2845.0 & 2859.6 & 9.9 & -4.7 & $2 \nu_{7}+\nu_{8}+\nu_{9}$ & $\nu_{6}+3 \nu_{7}$ \\
\hline 31 & $2858.76(0.84)$ & 2851.7 & 2866.3 & 7.1 & -7.5 & no & $2 \nu_{7}+\nu_{8}+\nu_{9}$ \\
\hline 32 & $2896.86(0.83)$ & 2857.0 & 2903.7 & 39.9 & -6.8 & no & $\nu_{5}+2 \nu_{9}$ \\
\hline 33 & $2900.37(0.70)$ & 2879.4 & 2926.5 & 21.0 & -26.1 & $\nu_{3}$ & $\nu_{5}+\nu_{6}+\nu_{7}$ \\
\hline 34 & $2923.67(0.66)$ & 2900.3 & 2946.8 & 23.4 & -23.1 & no & $\nu_{3}$ \\
\hline 35 & $2944.71(0.48)$ & 2912.1 & 2960.0 & 32.6 & -15.3 & no & $3 \nu_{6}$ \\
\hline
\end{tabular}


TABLE III - continued from previous page

\begin{tabular}{|c|c|c|c|c|c|c|c|}
\hline 36 & $2959.17(0.94)$ & 2941.8 & 2976.8 & 17.4 & -17.6 & $4 \nu_{9}$ & $\nu_{4}+\nu_{5}$ \\
\hline 37 & $2973.16(0.65)$ & 2956.4 & 3001.6 & 16.8 & -28.4 & no & $4 \nu_{9}$ \\
\hline 38 & $3000.33(0.84)$ & 2995.6 & 3022.5 & 4.7 & -22.2 & yes & $\nu_{5}+\nu_{8}+\nu_{9}$ \\
\hline 39 & $3018.11(0.85)$ & 3018.5 & 3069.9 & -0.4 & -51.8 & yes & $\nu_{2}$ \\
\hline 40 & $3038.39(0.93)$ & 3034.5 & 3076.6 & 3.9 & -38.2 & yes & $2 \nu_{7}+2 \nu_{8}$ \\
\hline 41 & $3069.84(0.66)$ & 3052.4 & 3106.6 & 17.4 & -36.8 & $\nu_{4}+2 \nu_{9}$ & $\nu_{6}+\nu_{7}+2 \nu_{9}$ \\
\hline 42 & $3074.82(0.77)$ & 3069.8 & 3120.5 & 5.0 & -45.7 & yes & $\nu_{4}+2 \nu_{9}$ \\
\hline 43 & $3121.35(0.82)$ & 3115.8 & 3132.9 & 5.5 & -11.6 & $\nu_{1}$ & $\nu_{4}+\nu_{6}+\nu_{7}$ \\
\hline 44 & $3128.62(0.82)$ & 3121.1 & 3133.4 & 7.5 & -4.8 & $\nu_{5}+2 \nu_{8}$ & $\nu_{1}$ \\
\hline 45 & $3135.34(0.89)$ & 3122.6 & 3142.2 & 12.7 & -6.9 & $\nu_{8}+3 \nu_{9}$ & $\nu_{5}+2 \nu_{8}$ \\
\hline 46 & $3142.11(0.81)$ & 3133.4 & 3159.1 & 8.7 & -17.0 & $\nu_{4}+\nu_{6}+\nu_{7}$ & $\nu_{8}+3 \nu_{9}$ \\
\hline 47 & $3160.52(0.61)$ & 3159.1 & 3159.9 & 1.4 & 0.6 & yes & $2 \nu_{4}$ \\
\hline 48 & $3208.31(0.82)$ & & & & & $\nu_{5}+3 \nu_{7}$ & \\
\hline 49 & $3217.43(0.74)$ & & & & & $\nu_{4}+\nu_{8}+\nu_{9}$ & \\
\hline$\cdots$ & $\cdots$ & $\cdots$ & $\cdots$ & $\cdots$ & $\cdots$ & $\cdots$ & $\cdots$ \\
\hline 65 & $3563.56(0.78)$ & & & & & $\nu_{3}+\nu_{7}$ & \\
\hline 67 & $3568.46(0.95)$ & & & & & $4 \nu_{8}$ & \\
\hline 75 & $3687.17(0.85)$ & & & & & $\nu_{2}+\nu_{7}$ & \\
\hline
\end{tabular}


TABLE IV: Same as Table III but for $B+\left(B_{2}\right)$ symmetry.

\begin{tabular}{|c|c|c|c|c|c|c|c|}
\hline$i$ & Cal1 & $\mathrm{Cal} 2$ & Cal3 & $\Delta_{12}$ & $\Delta_{13}$ & Assign1 & Assign2 \\
\hline 1 & $0.59(0.98)$ & .6 & .53 & 0.0 & 0.1 & yes & GS \\
\hline 2 & $681.56(0.97)$ & 679.6 & 681.4 & 2.0 & 0.2 & yes & $\nu_{7}$ \\
\hline 3 & $1009.99(0.98)$ & 1005.1 & 1009.8 & 4.9 & 0.2 & yes & $\nu_{6}$ \\
\hline 4 & $1356.86(0.68)$ & 1350.5 & 1356.4 & 6.4 & 0.5 & yes & $2 \nu_{7}$ \\
\hline 5 & $1359.50(0.67)$ & 1357.8 & 1359.4 & 1.7 & 0.1 & yes & $\nu_{5}$ \\
\hline 6 & $1505.16(0.98)$ & 1504.7 & 1505.0 & 0.5 & 0.2 & yes & $2 \nu \nu_{9}$ \\
\hline 7 & $1584.23(0.83)$ & 1582.8 & 1584.4 & 1.4 & -0.2 & yes & $\nu_{4}$ \\
\hline 8 & $1647.68(0.95)$ & 1640.6 & 1647.8 & 7.1 & -0.1 & yes & $\nu_{6}+\nu_{7}$ \\
\hline 9 & $1659.51(0.95)$ & 1651.4 & 1659.2 & 8.1 & 0.3 & yes & $\nu_{8}+\nu_{9}$ \\
\hline 10 & $1782.82(0.98)$ & 1782.1 & 1782.5 & 0.7 & 0.3 & yes & $2 \nu_{8}$ \\
\hline 11 & $1968.54(0.96)$ & 1948.1 & 1968.6 & 20.4 & -0.1 & no & $\left(\nu_{6}, \nu_{7}\right)$ \\
\hline 12 & $2039.64(0.84)$ & 2024.0 & 2039.8 & 15.6 & -0.2 & yes & $\nu_{5}+\nu_{7}$ \\
\hline 13 & $2043.53(0.95)$ & 2034.3 & 2043.5 & 9.2 & 0.0 & $3 \nu_{7}$ & $\left(\nu_{6}, \nu_{7}\right)$ \\
\hline 14 & $2182.79(0.96)$ & 2179.2 & 2182.7 & 3.6 & 0.1 & yes & $\nu_{7}+2 \nu_{9}$ \\
\hline 15 & $2260.97(0.81)$ & 2254.4 & 2260.9 & 6.6 & 0.1 & yes & $\nu_{4}+\nu_{7}$ \\
\hline 16 & $2328.51(0.93)$ & 2315.6 & 2328.5 & 12.9 & 0.0 & $\nu_{6}+2 \nu_{7}$ & $\left(\nu_{6}, \nu_{7}\right)$ \\
\hline 17 & $2340.48(0.90)$ & 2330.8 & 2340.6 & 9.7 & -0.1 & no & $\nu_{7}+\nu_{8}+\nu_{9}$ \\
\hline 18 & $2362.93(0.85)$ & 2344.7 & 2364.6 & 18.2 & -1.7 & yes & $\nu_{5}+\nu_{6}$ \\
\hline 19 & $2454.31(0.93)$ & 2415.3 & 2455.5 & 39.0 & -1.2 & no & $\nu_{6}+3 \nu_{7}$ \\
\hline 20 & $2476.47(0.95)$ & 2469.4 & 2477.1 & 7.1 & -0.6 & yes & $\nu_{7}+2 \nu_{8}$ \\
\hline 21 & $2512.73(0.97)$ & 2505.6 & 2513.2 & 7.1 & -0.5 & yes & $\nu_{6}+2 \nu_{9}$ \\
\hline 22 & $2583.97(0.84)$ & 2576.7 & 2556.6 & 7.3 & 27.4 & yes & $\nu_{4}+\nu_{6}$ \\
\hline 23 & $2658.20(0.97)$ & 2650.5 & 2652.5 & 7.7 & 5.7 & yes & $\nu_{6}+\nu_{8}+\nu_{9}$ \\
\hline 24 & $2708.17(0.76)$ & 2685.0 & 2658.9 & 23.2 & 49.3 & $2 \nu_{5}$ & $\nu_{5}+2 \nu_{7}$ \\
\hline 25 & $2716.15(0.61)$ & 2701.9 & 2710.2 & 14.2 & 5.9 & $4 \nu_{7}^{\dagger}$ & $2 \nu_{5}$ \\
\hline 26 & $2745.70(0.70)$ & 2709.0 & 2717.9 & 36.7 & 27.8 & $4 \nu_{7}^{\dagger}$ & $\left(\nu_{6}, \nu_{7}\right)$ \\
\hline 27 & $2797.81(0.91)$ & 2788.2 & 2747.3 & 9.6 & 50.5 & $\nu_{6}+2 \nu_{8}$ & $\nu_{6}+2 \nu_{8}$ \\
\hline 28 & $2860.29(0.79)$ & 2846.5 & 2799.0 & 13.8 & 61.3 & yes & $2 \nu_{7}+2 \nu_{9}$ \\
\hline 29 & $2865.26(0.69)$ & 2857.6 & 2861.7 & 7.7 & 3.6 & no & $\nu_{5}+2 \nu_{9}$ \\
\hline 30 & $2900.42(0.75)$ & 2891.4 & 2899.8 & 9.0 & 0.6 & $\nu_{3}$ & $\left(\nu_{6}, \nu_{7}\right)$ \\
\hline 31 & $2928.23(0.70)$ & 2898.9 & 2903.9 & 29.3 & 24.3 & $\nu_{4}+2 \nu_{7}$ & $\nu_{3}$ \\
\hline 32 & $2945.87(0.44)$ & 2917.9 & 2928.8 & 28.0 & 17.1 & no & $\nu_{4}+2 \nu_{7}$ \\
\hline 33 & $2963.72(0.91)$ & 2941.9 & 2947.9 & 21.8 & 15.8 & no & $\nu_{4}+\nu_{5}$ \\
\hline 34 & $2968.92(0.96)$ & 2962.8 & 2966.8 & 6.1 & 2.1 & $4 \nu_{9}$ & $4 \nu_{9}$ \\
\hline 35 & $2999.71(0.79)$ & 2964.0 & 2969.9 & 35.7 & 29.8 & no & $\nu_{5}+\nu_{6}+\nu_{7}$ \\
\hline 36 & $3010.62(0.81)$ & 2996.3 & 3002.5 & 14.3 & 8.1 & $2 \nu_{7}+\nu_{8}+\nu_{9}$ & $\nu_{5}+\nu_{8}+\nu_{9}$ \\
\hline 37 & $3013.58(0.72)$ & 3002.5 & 3012.9 & 11.1 & 0.7 & no & $2 \nu_{7}+\nu_{8}+\nu_{9}$ \\
\hline 38 & $3017.76(0.84)$ & 3018.2 & 3016.5 & -0.4 & 1.3 & $\nu_{2}$ & $\nu_{2}$ \\
\hline 39 & $3074.54(0.81)$ & 3042.8 & 3022.9 & 31.7 & 51.6 & $\nu_{4}+2 \nu_{9}$ & $\left(\nu_{6}, \nu_{7}\right)$ \\
\hline 40 & $3103.32(0.64)$ & 3072.0 & 3074.8 & 31.3 & 28.5 & no & $\nu_{4}+2 \nu_{9}$ \\
\hline 41 & $3120.58(0.69)$ & 3098.6 & 3121.1 & 22.0 & -0.5 & $\nu_{1}$ & $\left(\nu_{6}, \nu_{7}\right)$ \\
\hline 42 & $3124.82(0.71)$ & 3121.7 & 3127.2 & 3.1 & -2.4 & no & $\nu_{1}$ \\
\hline
\end{tabular}


TABLE IV - continued from previous page

\begin{tabular}{rrrrrrrr}
\hline \hline 43 & $3130.07(0.82)$ & 3122.6 & 3131.4 & 7.5 & -1.3 & yes & $\nu_{5}+2 \nu_{8}$ \\
44 & $3140.30(0.79)$ & 3136.3 & 3142.8 & 4.0 & -2.5 & no & $\nu_{8}+3 \nu_{9}$ \\
45 & $3159.54(0.84)$ & 3151.7 & 3160.9 & 7.8 & -1.4 & yes & $2 \nu_{7}+2 \nu_{8}$ \\
46 & $3160.43(0.57)$ & 3156.0 & & 4.4 & & $2 \nu_{4}$ & $2 \nu_{4}$ \\
47 & $3175.35(0.76)$ & & & & & $\nu_{4}+\nu_{6}+\nu_{7}$ & \\
48 & $3218.01(0.76)$ & & & & & & \\
49 & $3235.38(0.78)$ & & & & & $\nu_{4}+\nu_{8}+\nu_{9}$ & \\
50 & $3294.92(0.92)$ & & & & & $\ldots$ & $\ldots$ \\
$\ldots$ & $\ldots$ & $\ldots$ & $\ldots$ & $\ldots$ & $\ldots$ & & \\
64 & $3571.99(0.88)$ & & & & & & \\
63 & $3574.46(0.73)$ & & & & & $\nu_{3}+\nu_{7}$ & \\
70 & $3676.12(0.75)$ & & & & & $\nu_{2}+\nu_{7}$ & \\
\hline \hline
\end{tabular}

† The first two major components of both levels are the same and both components look like $4 \nu_{7}$. 
TABLE V: Same as Table III but for $A-\left(B_{1}\right)$ symmetry.

\begin{tabular}{|c|c|c|c|c|c|c|c|}
\hline$i$ & Cal1 & $\mathrm{Cal} 2$ & Cal3 & $\Delta_{12}$ & $\Delta_{13}$ & Assign1 & Assign2 \\
\hline 1 & $755.72(0.98)$ & 755.7 & 755.1 & 0.0 & 0.6 & yes & $\nu_{9}$ \\
\hline 2 & $889.98(0.98)$ & 889.7 & 889.7 & 0.3 & 0.3 & yes & $\nu_{8}$ \\
\hline 3 & $1405.40(0.96)$ & 1403.9 & 1405.0 & 1.5 & 0.4 & yes & $\nu_{7}+\nu_{9}$ \\
\hline 4 & $1560.82(0.96)$ & 1558.3 & 1560.4 & 2.5 & 0.4 & yes & $\nu_{7}+\nu_{8}$ \\
\hline 5 & $1738.05(0.97)$ & 1732.7 & 1737.5 & 5.4 & 0.6 & yes & $\nu_{6}+\nu_{9}$ \\
\hline 6 & $1889.26(0.97)$ & 1882.3 & 1888.9 & 7.0 & 0.4 & yes & $\nu_{6}+\nu_{8}$ \\
\hline 7 & $1957.44(0.95)$ & 1956.3 & 1957.0 & 1.1 & 0.4 & yes & $2 \nu_{7}+\nu_{9}$ \\
\hline 8 & $2113.57(0.85)$ & 2110.5 & 2112.3 & 3.1 & 1.3 & yes & $\nu_{5}+\nu_{9}$ \\
\hline 9 & $2141.69(0.95)$ & 2139.1 & 2141.5 & 2.6 & 0.2 & yes & $2 \nu_{7}+\nu_{8}$ \\
\hline 10 & $2236.65(0.95)$ & 2235.5 & 2236.0 & 1.2 & 0.7 & yes & $3 \nu 9$ \\
\hline 11 & $2243.10(0.84)$ & 2239.2 & 2243.4 & 3.9 & -0.3 & yes & $\nu_{5}+\nu_{8}$ \\
\hline 12 & $2320.81(0.95)$ & 2305.6 & 2320.6 & 15.2 & 0.2 & yes & $\nu_{6}+\nu_{7}+\nu_{9}$ \\
\hline 13 & $2332.31(0.83)$ & 2331.4 & 2331.3 & 0.9 & 1.0 & yes & $\nu_{4}+\nu_{9}$ \\
\hline 14 & $2396.60(0.96)$ & 2395.6 & 2396.1 & 1.0 & 0.5 & yes & $\nu_{8}+2 \nu_{9}$ \\
\hline 15 & $2466.21(0.65)$ & 2447.1 & 2466.0 & 19.1 & 0.2 & no & $\nu_{6}+\nu_{7}+\nu_{8}$ \\
\hline 16 & $2467.14(0.70)$ & 2466.0 & 2467.4 & 1.1 & -0.3 & no & $\nu_{4}+\nu_{8}$ \\
\hline 17 & $2540.93(0.96)$ & 2540.0 & 2540.4 & 0.9 & 0.5 & yes & $2 \nu_{8}+\nu_{9}$ \\
\hline 18 & $2608.39(0.94)$ & 2599.1 & 2608.8 & 9.3 & -0.4 & yes & $3 \nu_{7}+\nu_{9}$ \\
\hline 19 & $2674.50(0.97)$ & 2673.0 & 2674.6 & 1.5 & -0.1 & yes & $3 \nu_{8}$ \\
\hline 20 & $2749.07(0.82)$ & 2735.5 & 2749.8 & 13.6 & -0.7 & yes & $3 \nu_{7}+\nu_{8}$ \\
\hline 21 & $2761.72(0.62)$ & 2745.5 & 2762.9 & 16.2 & -1.2 & yes & $2 \nu_{6}+\nu_{9}$ \\
\hline 22 & $2765.96(0.66)$ & 2754.0 & 2767.1 & 12.0 & -1.1 & yes & $\nu_{5}+\nu_{7}+\nu_{9}$ \\
\hline 23 & $2836.18(0.92)$ & 2834.3 & 2836.7 & 1.9 & -0.5 & yes & $\nu_{7}+3 \nu_{9}$ \\
\hline 24 & $2909.04(0.97)$ & 2883.3 & 2910.6 & 25.7 & -1.6 & yes & $2 \nu_{6}+\nu_{8}$ \\
\hline 25 & $2915.02(0.85)$ & 2901.5 & 2923.9 & 13.5 & -8.9 & yes & $\nu_{5}+\nu_{7}+\nu_{8}$ \\
\hline 26 & $2977.49(0.78)$ & 2958.1 & 2977.6 & 19.4 & -0.1 & $\nu_{4}+\nu_{7}+\nu_{9}$ & $\nu_{6}+2 \nu_{7}+\nu_{9}$ \\
\hline 27 & $2991.00(0.88)$ & 2975.3 & 3006.7 & 15.7 & -15.7 & $\nu_{6}+2 \nu_{7}+\nu_{9}$ & $\nu_{4}+\nu_{7}+\nu_{9}$ \\
\hline 28 & $3025.06(0.91)$ & 3022.3 & 3025.8 & 2.8 & -0.7 & no & $\nu_{7}+\nu_{8}+2 \nu_{9}$ \\
\hline 29 & $3091.37(0.85)$ & 3067.9 & 3103.4 & 23.5 & -12.0 & yes & $\nu_{5}+\nu_{6}+\nu_{9}$ \\
\hline 30 & $3121.63(0.88)$ & 3088.1 & 3118.4 & 33.5 & 3.2 & yes & $\nu_{6}+2 \nu_{7}+\nu_{8}$ \\
\hline 31 & $3136.21(0.77)$ & 3129.3 & 3136.7 & 6.9 & -0.5 & yes & $\nu_{4}+\nu_{7}+\nu_{8}$ \\
\hline 32 & $3183.09(0.96)$ & 3174.9 & 3197.7 & 8.2 & -14.6 & yes & $\nu_{6}+3 \nu_{9}$ \\
\hline 33 & $3198.30(0.95)$ & 3194.1 & 3200.5 & 4.2 & -2.2 & yes & $\nu_{7}+2 \nu_{8}+\nu_{9}$ \\
\hline 34 & $3237.48(0.86)$ & 3208.3 & & 29.2 & & yes & $\nu_{5}+\nu_{6}+\nu_{8}$ \\
\hline
\end{tabular}


TABLE VI: Same as Table III but for $B-\left(A_{2}\right)$ symmetry.

\begin{tabular}{|c|c|c|c|c|c|c|c|}
\hline$i$ & Cal1 & Cal2 & Cal3 & $\Delta_{12}$ & $\Delta_{13}$ & Assign1 & Assign2 \\
\hline 1 & $757.24(0.98)$ & 757.2 & 757.2 & 0.0 & 0.0 & yes & $\nu_{9}$ \\
\hline 2 & $890.62(0.98)$ & 890.3 & 890.4 & 0.3 & 0.2 & yes & $\nu_{8}$ \\
\hline 3 & $1435.38(0.97)$ & 1433.4 & 1435.0 & 2.0 & 0.4 & yes & $\nu_{7}+\nu_{9}$ \\
\hline 4 & $1575.87(0.97)$ & 1573.1 & 1575.5 & 2.8 & 0.4 & yes & $\nu_{7}+\nu_{8}$ \\
\hline 5 & $1763.34(0.98)$ & 1758.2 & 1762.8 & 5.1 & 0.5 & yes & $\nu_{6}+\nu_{9}$ \\
\hline 6 & $1902.84(0.97)$ & 1896.0 & 1902.5 & 6.8 & 0.3 & yes & $\nu_{6}+\nu_{8}$ \\
\hline 7 & $2112.59(0.96)$ & 2106.8 & 2113.5 & 5.8 & -0.9 & yes & $2 \nu_{7}+\nu_{9}$ \\
\hline 8 & $2115.16(0.85)$ & 2112.6 & 2115.5 & 2.6 & -0.3 & yes & $\nu_{5}+\nu_{9}$ \\
\hline 9 & $2241.72(0.88)$ & 2237.3 & 2241.6 & 4.4 & 0.1 & $3 \nu_{9}$ & $\nu_{5}+\nu_{8}$ \\
\hline 10 & $2243.34(0.78)$ & 2240.7 & 2243.3 & 2.6 & 0.0 & $\nu_{5}+\nu_{8}$ & $3 \nu_{9}$ \\
\hline 11 & $2256.96(0.95)$ & 2252.3 & 2256.8 & 4.7 & 0.2 & yes & $2 \nu_{7}+\nu_{8}$ \\
\hline 12 & $2333.40(0.83)$ & 2331.7 & 2333.9 & 1.7 & -0.5 & yes & $\nu_{4}+\nu_{9}$ \\
\hline 13 & $2399.77(0.95)$ & 2396.9 & 2399.7 & 2.9 & 0.1 & $\nu_{8}+2 \nu_{9}$ & $\nu_{6}+\nu_{7}+\nu_{9}$ \\
\hline 14 & $2412.32(0.94)$ & 2401.4 & 2412.1 & 10.9 & 0.2 & no & $\nu_{8}+2 \nu_{9}$ \\
\hline 15 & $2466.99(0.83)$ & 2464.8 & 2466.5 & 2.2 & 0.5 & yes & $\nu_{4}+\nu_{8}$ \\
\hline 16 & $2540.52(0.94)$ & 2531.3 & 2541.2 & 9.2 & -0.7 & $2 \nu_{8}+\nu_{9}$ & $\nu_{6}+\nu_{7}+\nu_{8}$ \\
\hline 17 & $2556.29(0.95)$ & 2546.0 & 2556.4 & 10.3 & -0.1 & no & $2 \nu_{8}+\nu_{9}$ \\
\hline 18 & $2676.59(0.95)$ & 2667.1 & 2676.8 & 9.5 & -0.2 & $3 \nu_{8}$ & $3 \nu_{7}+\nu_{9}$ \\
\hline 19 & $2688.79(0.94)$ & 2675.9 & 2689.7 & 12.9 & -0.9 & no & $3 \nu_{8}$ \\
\hline 20 & $2793.24(0.77)$ & 2780.7 & 2749.8 & 12.5 & 43.4 & yes & $\nu_{5}+\nu_{7}+\nu_{9}$ \\
\hline 21 & $2805.85(0.85)$ & 2792.2 & 2762.9 & 13.6 & 42.9 & yes & $2 \nu_{6}+\nu_{9}$ \\
\hline 22 & $2867.55(0.94)$ & 2840.9 & 2767.1 & 26.7 & 100.5 & yes & $3 \nu_{7}+\nu_{8}$ \\
\hline 23 & $2923.34(0.95)$ & 2911.1 & 2916.5 & 12.2 & 6.8 & $\nu_{7}+3 \nu_{9}$ & $\nu_{5}+\nu_{7}+\nu_{8}$ \\
\hline 24 & $2929.29(0.83)$ & 2920.0 & 2930.7 & 9.3 & -1.4 & $\nu_{5}+\nu_{7}$ & $+\nu_{8} \nu_{7}+3 \nu_{9}$ \\
\hline 25 & $2942.66(0.94)$ & 2929.6 & 2943.9 & 13.1 & -1.2 & no & $2 \nu_{6}+\nu_{8}$ \\
\hline 26 & $3006.47(0.80)$ & 3000.7 & 2992.4 & 5.8 & 14.1 & yes & $\nu_{4}+\nu_{7}+\nu_{9}$ \\
\hline 27 & $3080.85(0.95)$ & 3076.2 & 3081.5 & 4.6 & -0.7 & no & $\nu_{7}+\nu_{8}+2 \nu_{9}$ \\
\hline 28 & $3101.58(0.90)$ & 3088.1 & 3093.6 & 13.5 & 8.0 & no & $\nu_{5}+\nu_{6}+\nu_{9}$ \\
\hline 29 & $3116.05(0.84)$ & 3099.2 & 3123.8 & 16.8 & -7.8 & $\nu_{5}+\nu_{6}+\nu_{9}$ & $\nu_{6}+2 \nu_{7}+\nu_{9}$ \\
\hline 30 & $3147.95(0.81)$ & 3137.3 & 3148.2 & 10.7 & -0.2 & yes & $\nu_{4}+\nu_{7}+\nu_{8}$ \\
\hline 31 & $3195.11(0.93)$ & 3152.3 & & 42.8 & & no & $2 \nu_{6}+\nu_{7}+\nu_{9}$ \\
\hline 32 & $3230.45(0.87)$ & 3208.2 & & 22.3 & & $\nu_{7}+2 \nu_{8}+\nu_{9}$ & $\nu_{6}+2 \nu_{7}+\nu_{8}$ \\
\hline 33 & $3240.80(0.80)$ & 3224.0 & & 16.8 & & no & $\nu_{7}+2 \nu_{8}+\nu_{9}$ \\
\hline 34 & $3250.72(0.80)$ & 3234.7 & & 16.0 & & $\nu_{5}+\nu_{6}+\nu_{8}$ & $2 \nu_{6}+\nu_{7}+\nu_{8}$ \\
\hline 35 & $3255.16(0.77)$ & 3245.3 & & 9.9 & & yes & $\nu_{6}+3 \nu_{9}$ \\
\hline
\end{tabular}


TABLE VII: Selected high vibrational energy levels (in $\mathrm{cm}^{-1}$ ) of $\mathrm{C}_{2} \mathrm{H}_{3}$ matched by assignment. Cal1 is from this paper. Cal2 and Cal3 refer to calculated levels from Šmydke et al.[4] and Yu et al. [3], respectively. $\Delta_{12}=$ Cal1 - Cal2, $\Delta_{13}=$ Cal1 - Cal3.

\begin{tabular}{|c|c|c|c|c|c|c|c|c|c|c|}
\hline \multirow[t]{2}{*}{ state } & Cal1 & Cal2 & Cal3 & $\Delta_{12}$ & $\Delta_{13}$ & Cal1 & Cal2 & Cal3 & $\Delta_{12}$ & $\Delta_{13}$ \\
\hline & \multicolumn{5}{|c|}{$A+\left(A_{1}\right)$} & \multicolumn{5}{|c|}{$B+\left(B_{2}\right)$} \\
\hline$\nu_{4}+\nu_{6}$ & 2570.55 & 2563.9 & 2584.2 & 6.6 & -13.7 & 2583.97 & 2576.7 & 2556.6 & 7.3 & 27.4 \\
\hline $2 \nu_{5}$ & 2709.26 & 2696.9 & 2710.4 & 12.4 & -1.1 & 2708.17 & 2701.9 & 2710.2 & 6.3 & -2.0 \\
\hline$\nu_{6}+2 \nu_{8}$ & 2783.62 & 2773.9 & 2784.8 & 9.7 & -1.2 & 2797.81 & 2788.2 & 2799.0 & 9.6 & -1.2 \\
\hline$\nu_{4}+2 \nu_{7}$ & 2828.16 & 2819.1 & 2828.5 & 9.1 & -0.3 & 2928.23 & 2917.9 & 2899.8 & 10.3 & 28.4 \\
\hline$\nu_{3}$ & 2900.37 & 2900.3 & 2903.7 & 0.1 & -3.3 & 2900.42 & 2898.9 & 2903.9 & 1.5 & -3.5 \\
\hline $4 \nu_{9}$ & 2959.17 & 2956.4 & 2960.0 & 2.8 & -0.8 & 2968.92 & 2962.8 & 2969.9 & 6.1 & -1.0 \\
\hline$\nu_{2}$ & 3018.11 & 3018.5 & 3022.5 & -0.4 & -4.4 & 3017.76 & 3018.2 & 3022.9 & -0.4 & -5.1 \\
\hline$\nu_{4}+2 \nu_{9}$ & 3069.84 & 3069.8 & 3069.9 & 0.0 & -0.1 & 3074.54 & 3072.0 & 3074.8 & 2.5 & -0.3 \\
\hline$\nu_{1}$ & 3121.35 & 3121.1 & 3120.5 & 0.2 & 0.8 & 3120.58 & 3121.7 & 3121.1 & -1.1 & -0.5 \\
\hline \multirow[t]{2}{*}{$2 \nu_{4}$} & 3160.52 & 3159.1 & 3159.9 & 1.4 & 0.6 & 3160.43 & 3156.0 & 3160.9 & 4.4 & -0.5 \\
\hline & \multicolumn{5}{|c|}{$A-$} & \multicolumn{5}{|c|}{$B-$} \\
\hline $3 \nu_{9}$ & 2236.65 & 2235.5 & 2236.0 & 1.2 & 0.7 & 2241.72 & 2240.7 & 2241.6 & 1.0 & 0.1 \\
\hline $3 \nu_{8}$ & 2674.50 & 2673.0 & 2674.6 & 1.5 & -0.1 & 2676.59 & 2675.9 & 2676.8 & 0.7 & -0.2 \\
\hline$\nu_{4}+\nu_{7}+\nu_{9}$ & 2977.49 & 2975.3 & 2977.6 & 2.2 & -0.1 & 3006.47 & 3000.7 & 2992.4 & 5.8 & 14.1 \\
\hline$\nu_{4}+\nu_{7}+\nu_{8}$ & 3136.21 & 3129.3 & 3136.7 & 6.9 & -0.5 & 3147.95 & 3137.3 & 3148.2 & 10.7 & -0.2 \\
\hline
\end{tabular}


TABLE VIII: Tunnelling splittings (in $\mathrm{cm}^{-1}$ ) of the $\mathrm{C}_{2} \mathrm{H}_{3}$ fundamentals. Only $A+$ levels for the $A+/ B+$ tunnelling pair and $A-$ levels for the $A-/ B-$ tunnelling pair are shown. Call is this paper. Cal2 and Cal3 numbers are from S̆mydke et al.[4] and Yu et al.[3], respectively.

\begin{tabular}{lrrrr}
\hline \hline state & Cal1 & Cal2 & Cal3 & Expt. \\
\hline$\Delta$ GS & 0.59 & 0.59 & 0.53 & 0.5428 \\
$\nu_{1}$ & 3121.35 & 3121.12 & 3120.46 & \\
$\Delta \nu_{1}$ & -0.77 & 0.56 & 0.68 & \\
$\nu_{2}$ & 3018.11 & 3018.49 & 3022.53 & \\
$\Delta \nu_{2}$ & -0.35 & -0.25 & 0.36 & \\
$\nu_{3}$ & 2900.37 & 2900.26 & 2903.70 & 2901.8603 \\
$\Delta \nu_{3}$ & 0.05 & -1.33 & 0.19 & 0.6144 \\
$\nu_{4}$ & 1583.88 & 1583.26 & 1583.59 & \\
$\Delta \nu_{4}$ & 0.36 & -0.51 & 0.76 & \\
$\nu_{5}$ & 1358.13 & 1355.24 & 1357.82 & \\
$\Delta \nu_{5}$ & 1.37 & 2.59 & 1.59 & \\
$\nu_{6}$ & 996.44 & 991.43 & 996.27 & \\
$\Delta \nu_{6}$ & 13.55 & 13.69 & 13.55 & \\
$\nu_{7}$ & 667.66 & 665.89 & 667.48 & \\
$\Delta \nu_{7}$ & 13.90 & 13.68 & 13.91 & \\
$\nu_{8}$ & 889.98 & 889.70 & 889.72 & 895.1625 \\
$\Delta \nu_{8}$ & 0.64 & 0.64 & 0.65 & 0.5985 \\
$\nu_{9}$ & 755.72 & 755.66 & 755.14 & \\
$\Delta \nu_{9}$ & 1.51 & 1.51 & 2.08 & \\
\hline \hline
\end{tabular}


(H)

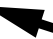

$\mathrm{q}_{1}$

$q_{0}$

$\mathrm{H}_{2}$

$\mathrm{q}_{2}$

C

$q_{3}$ 


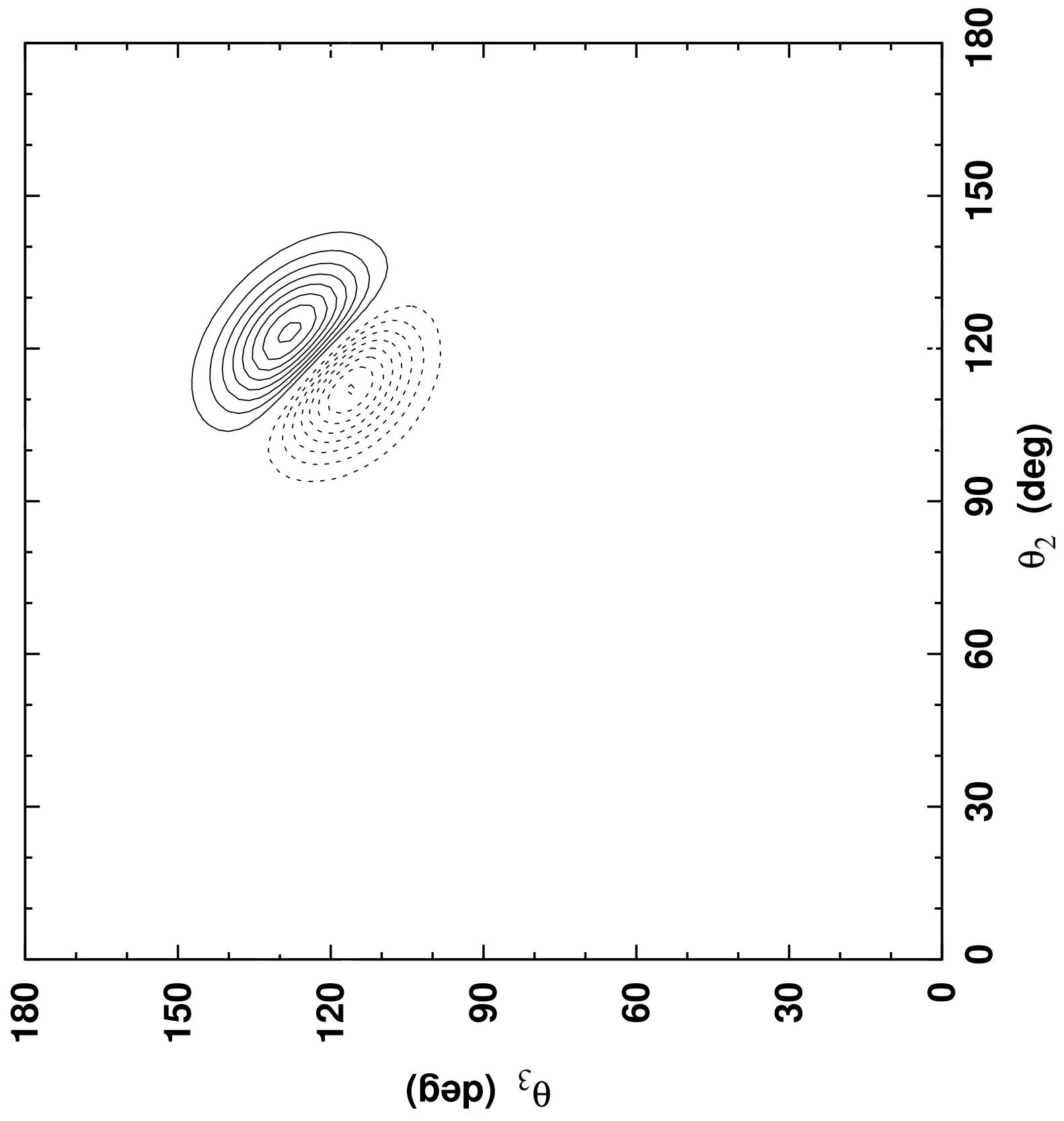




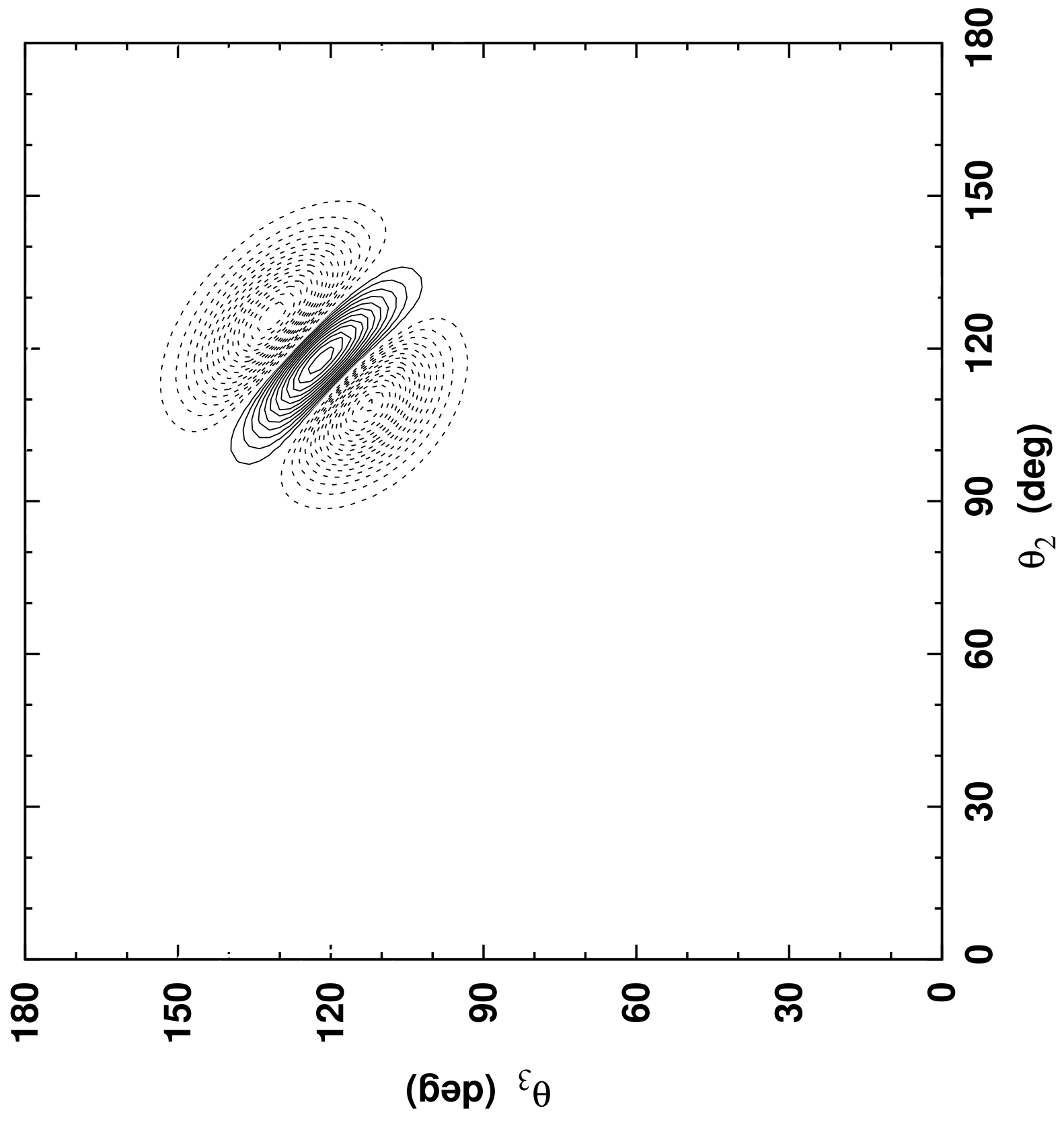




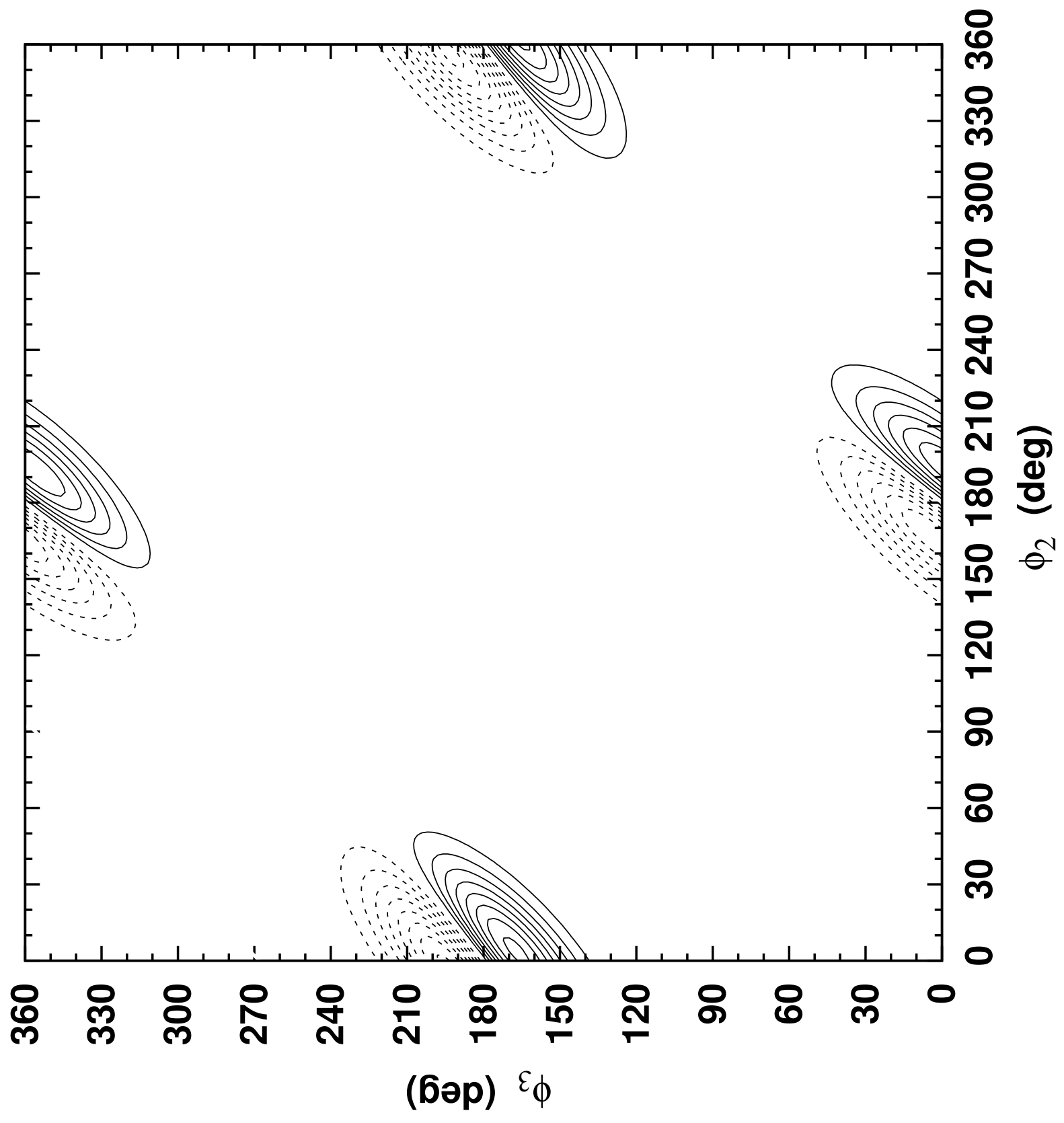




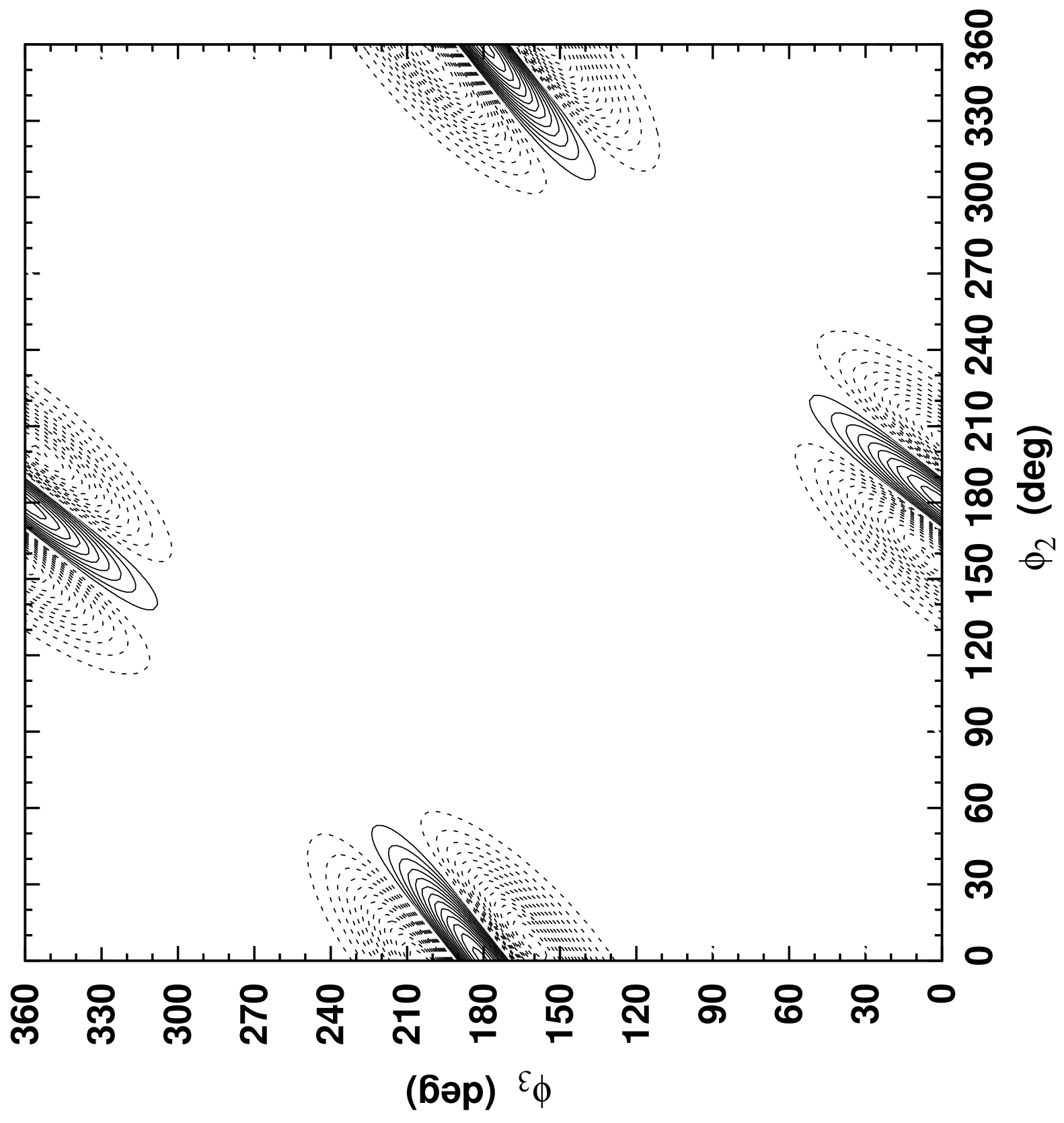




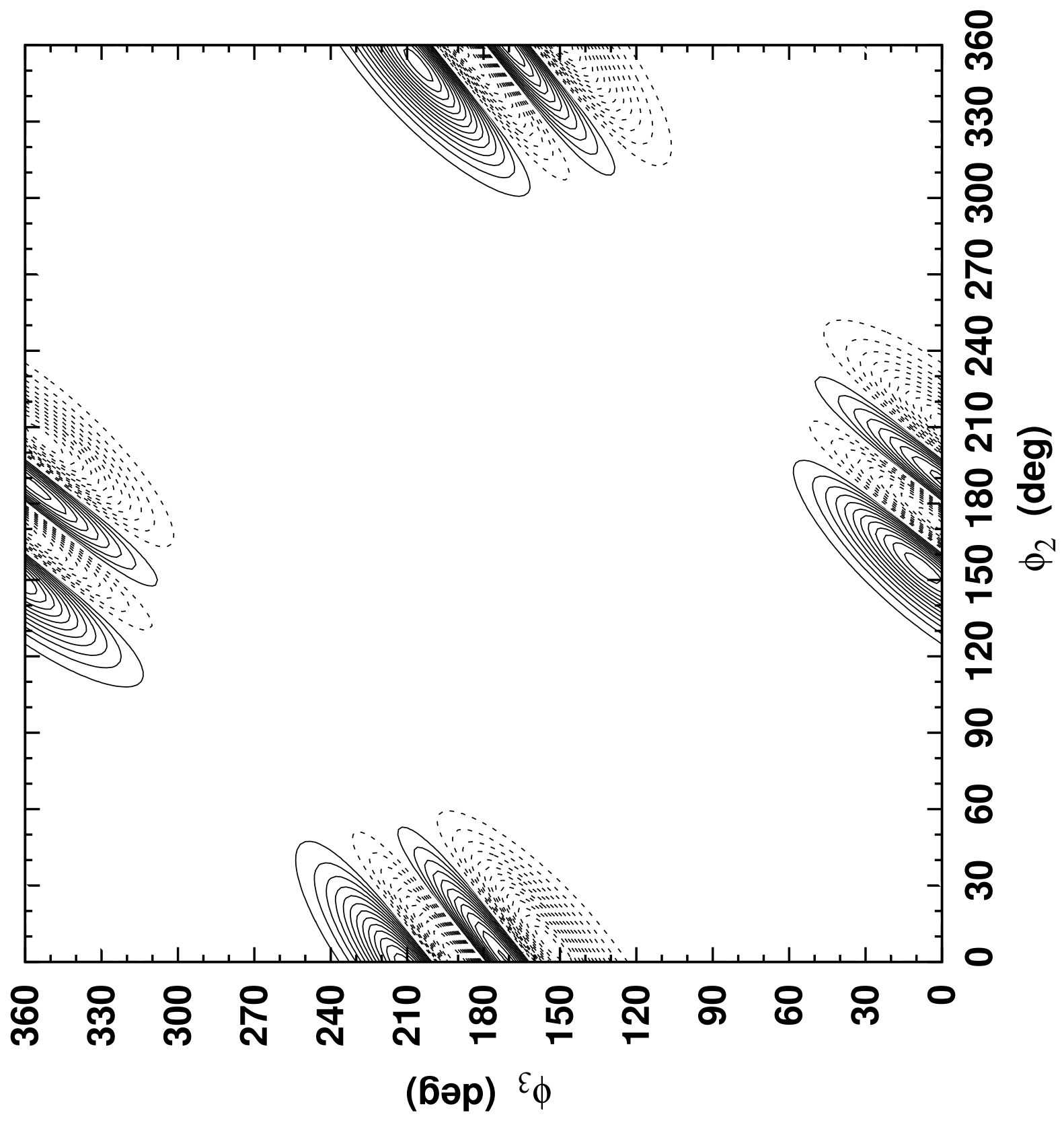




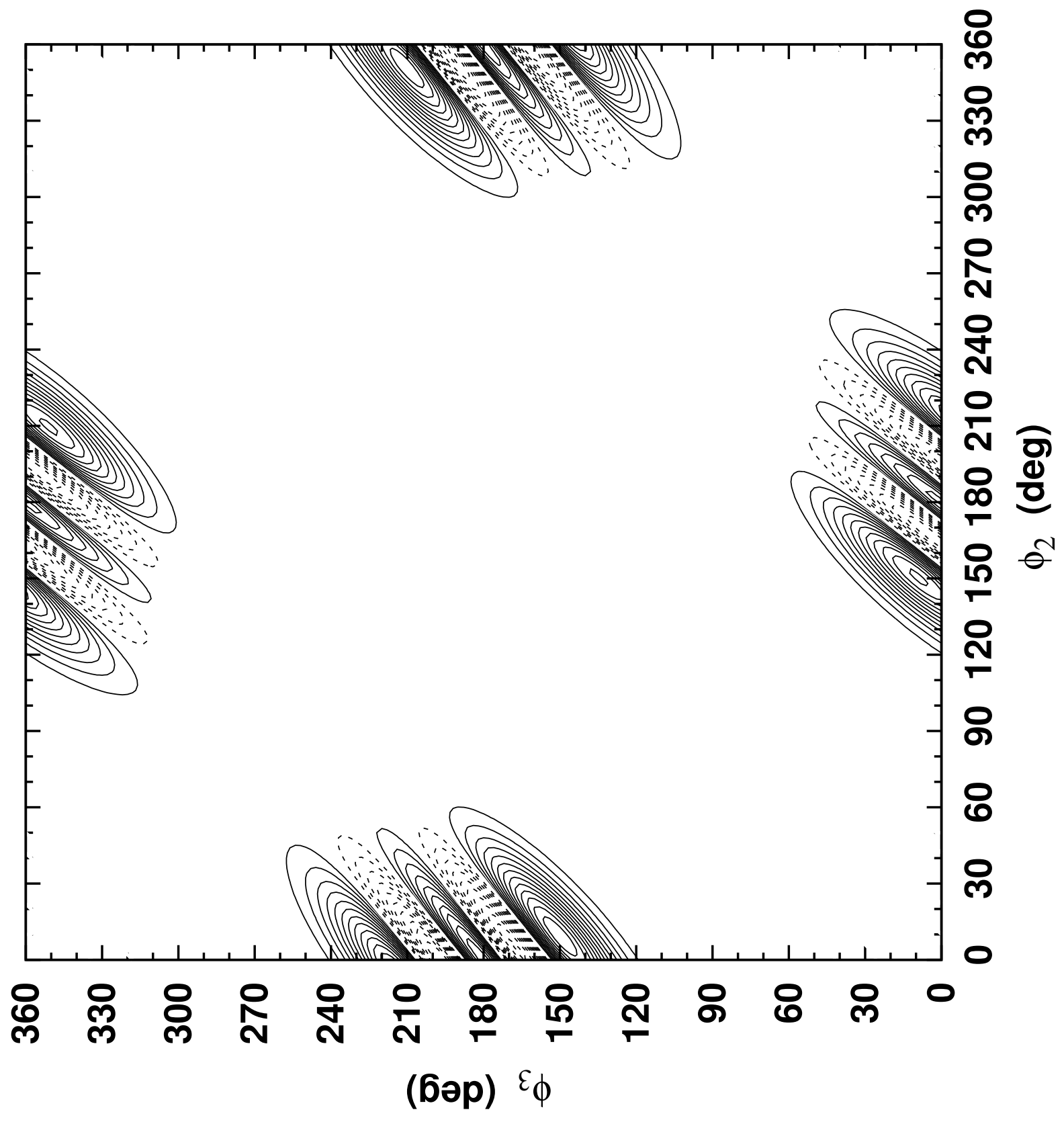




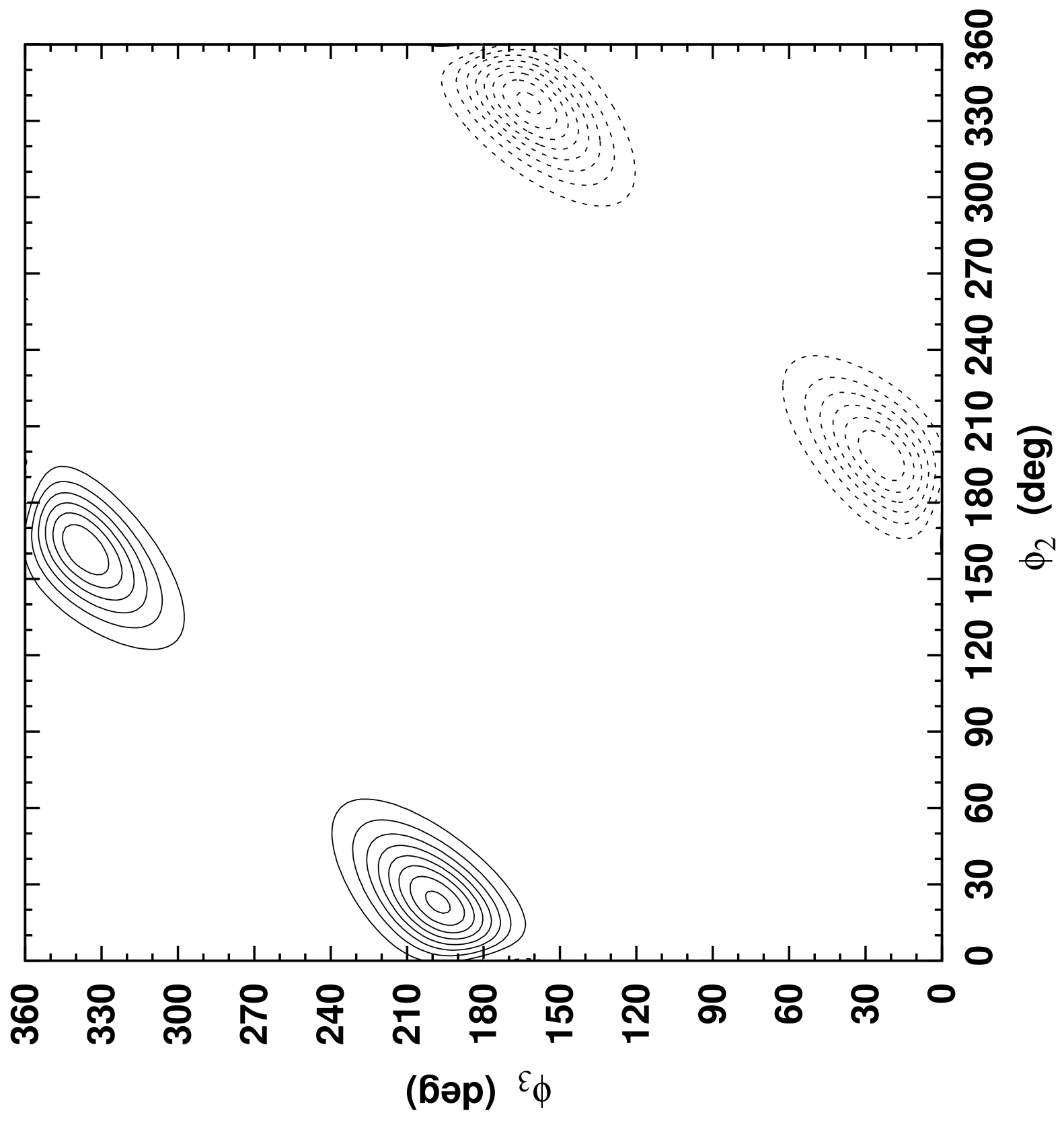




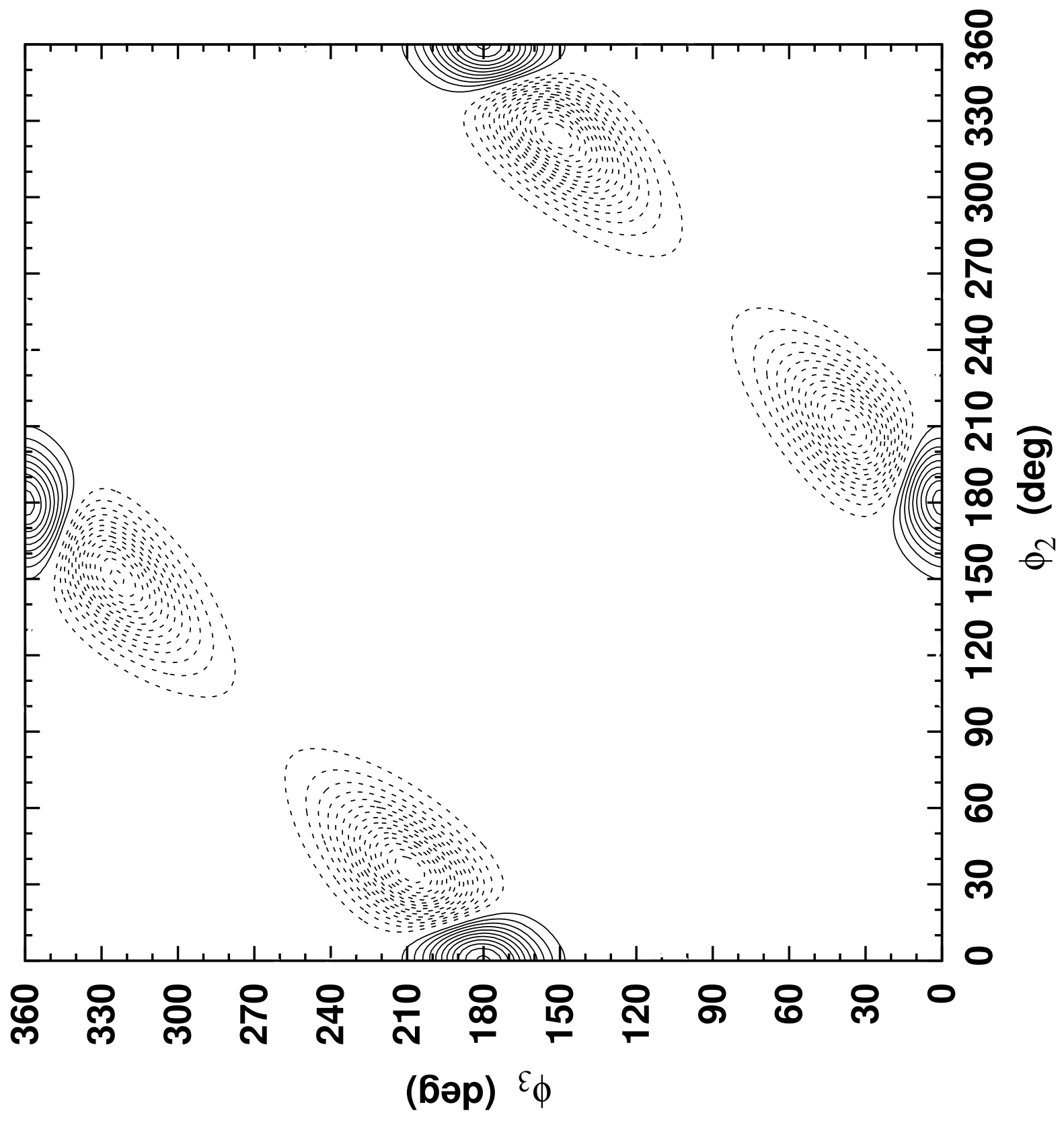




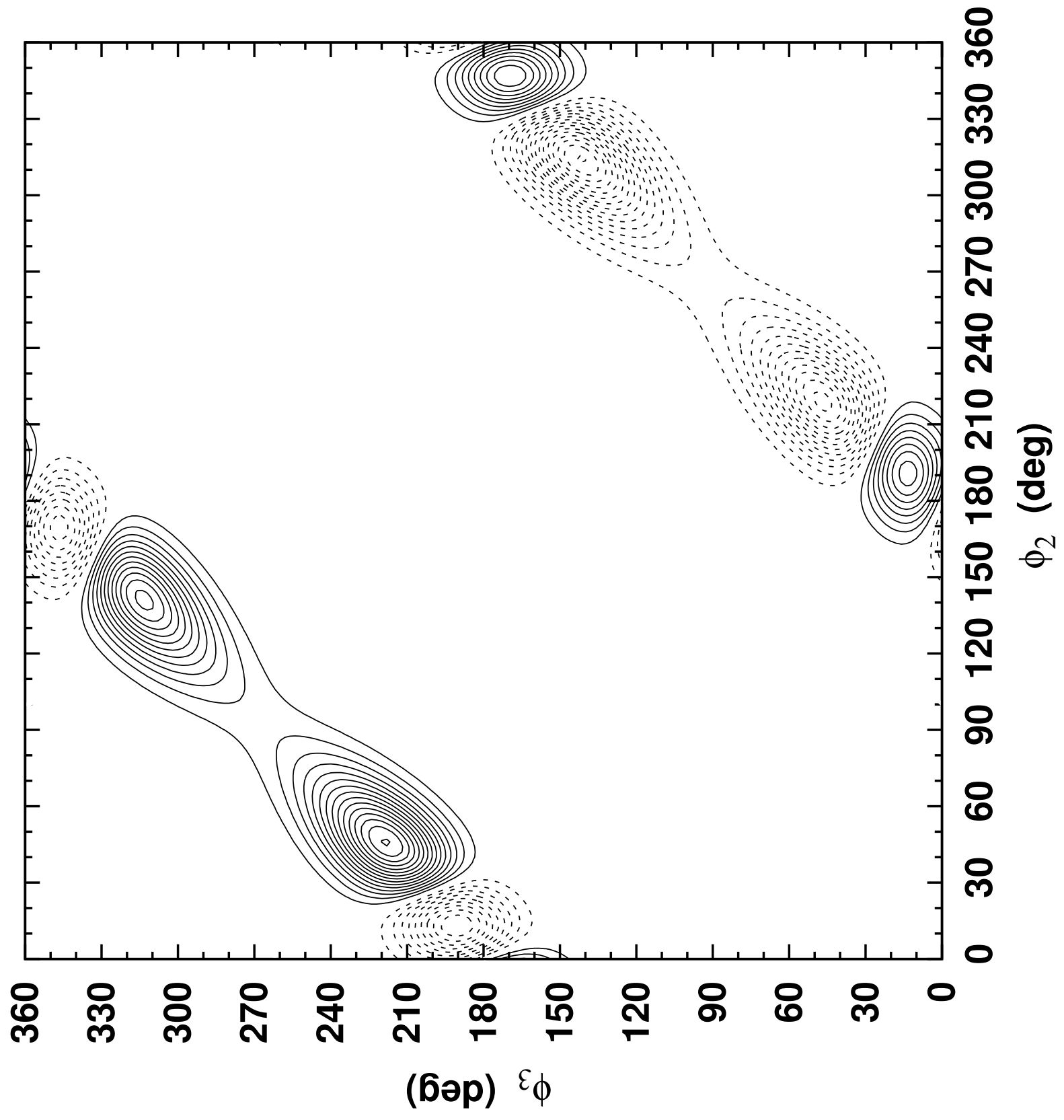




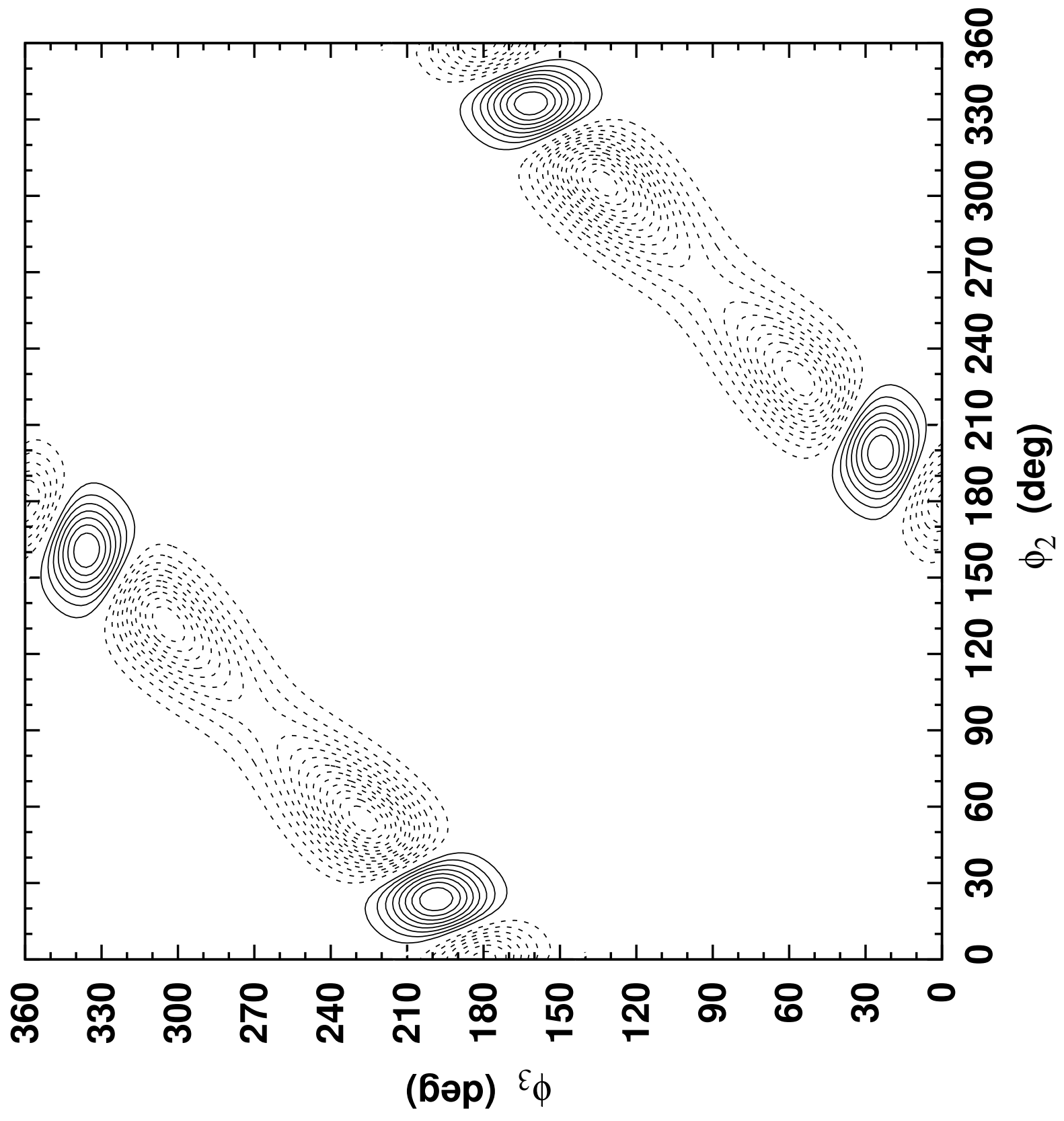




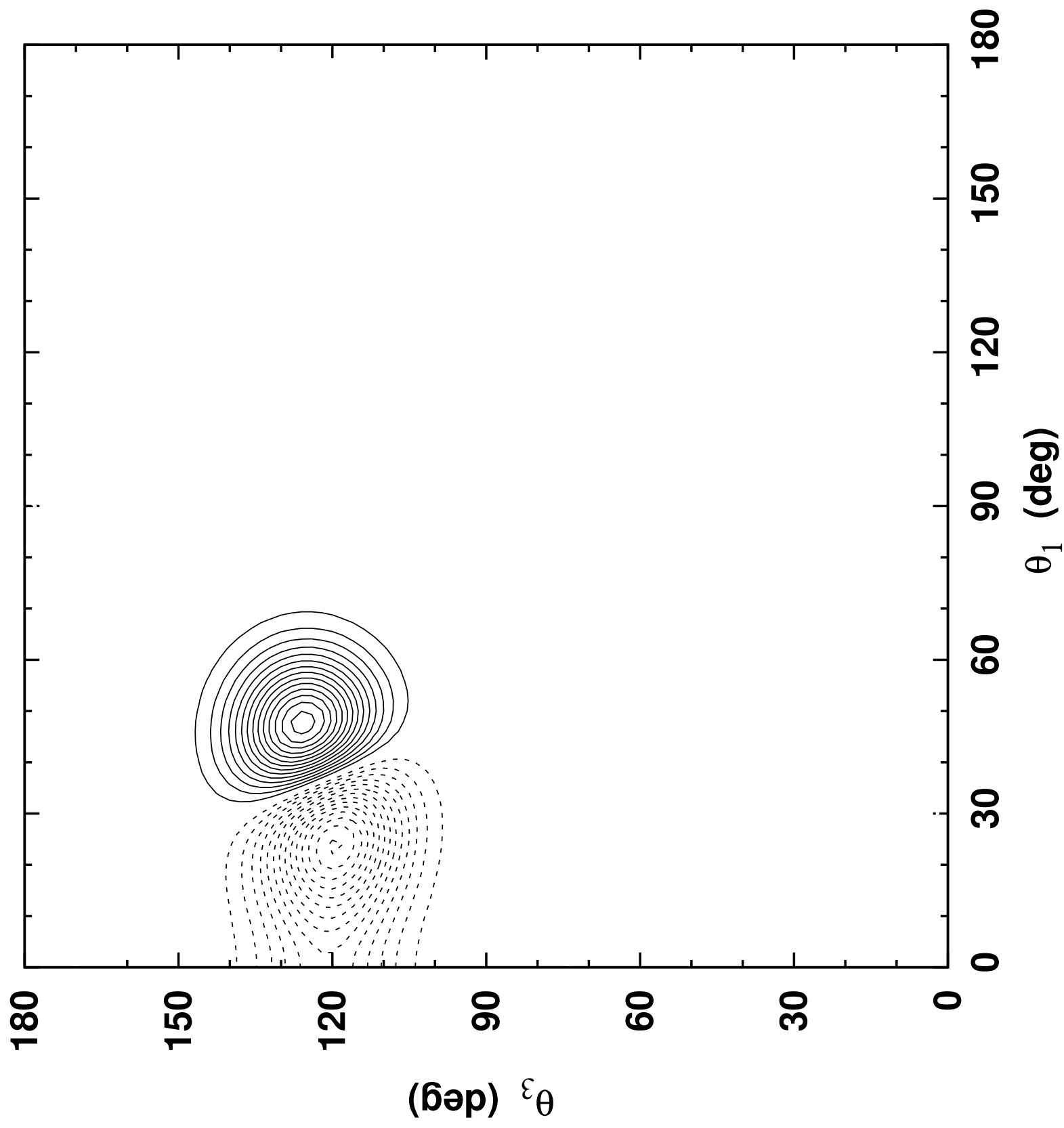




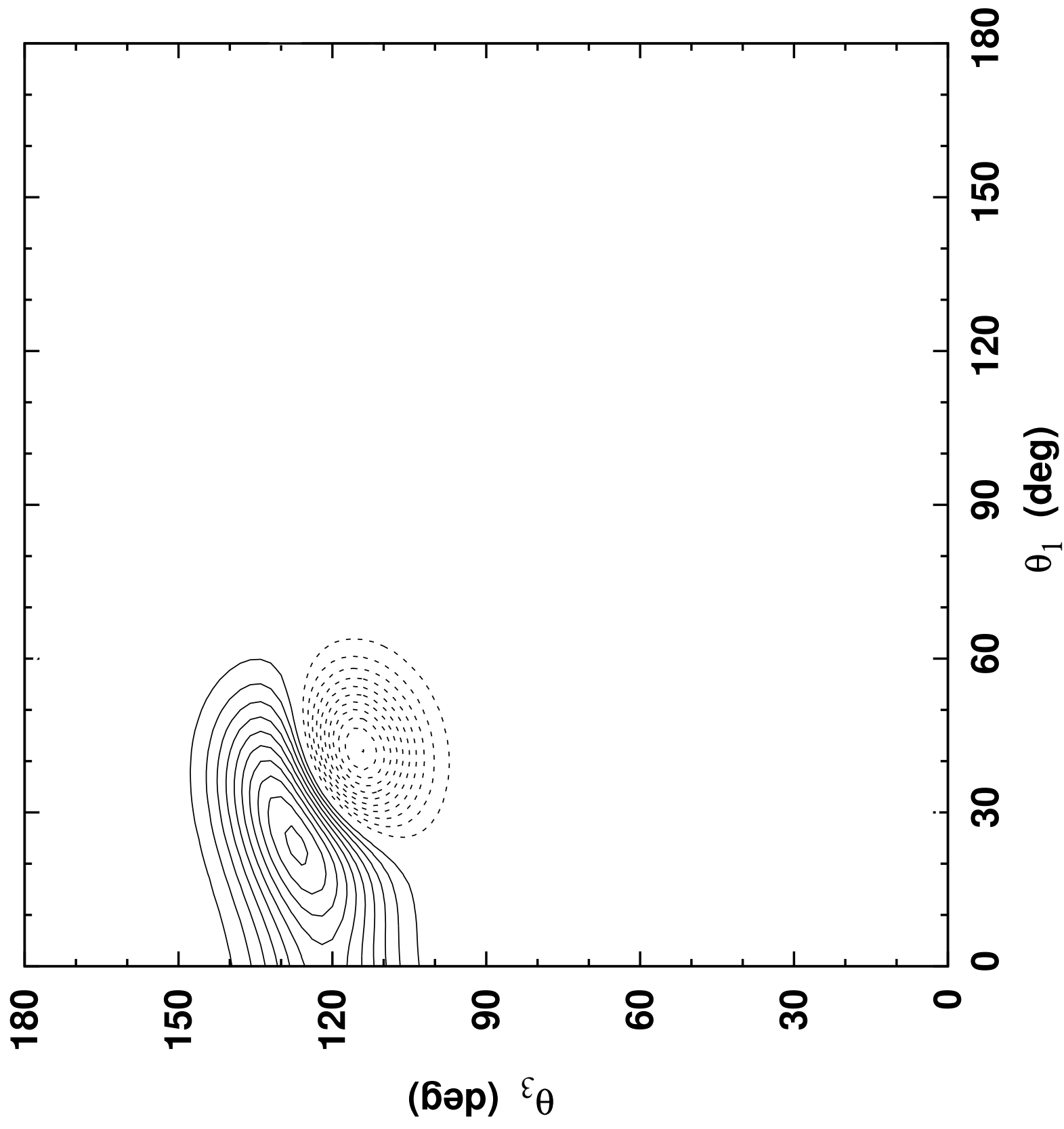




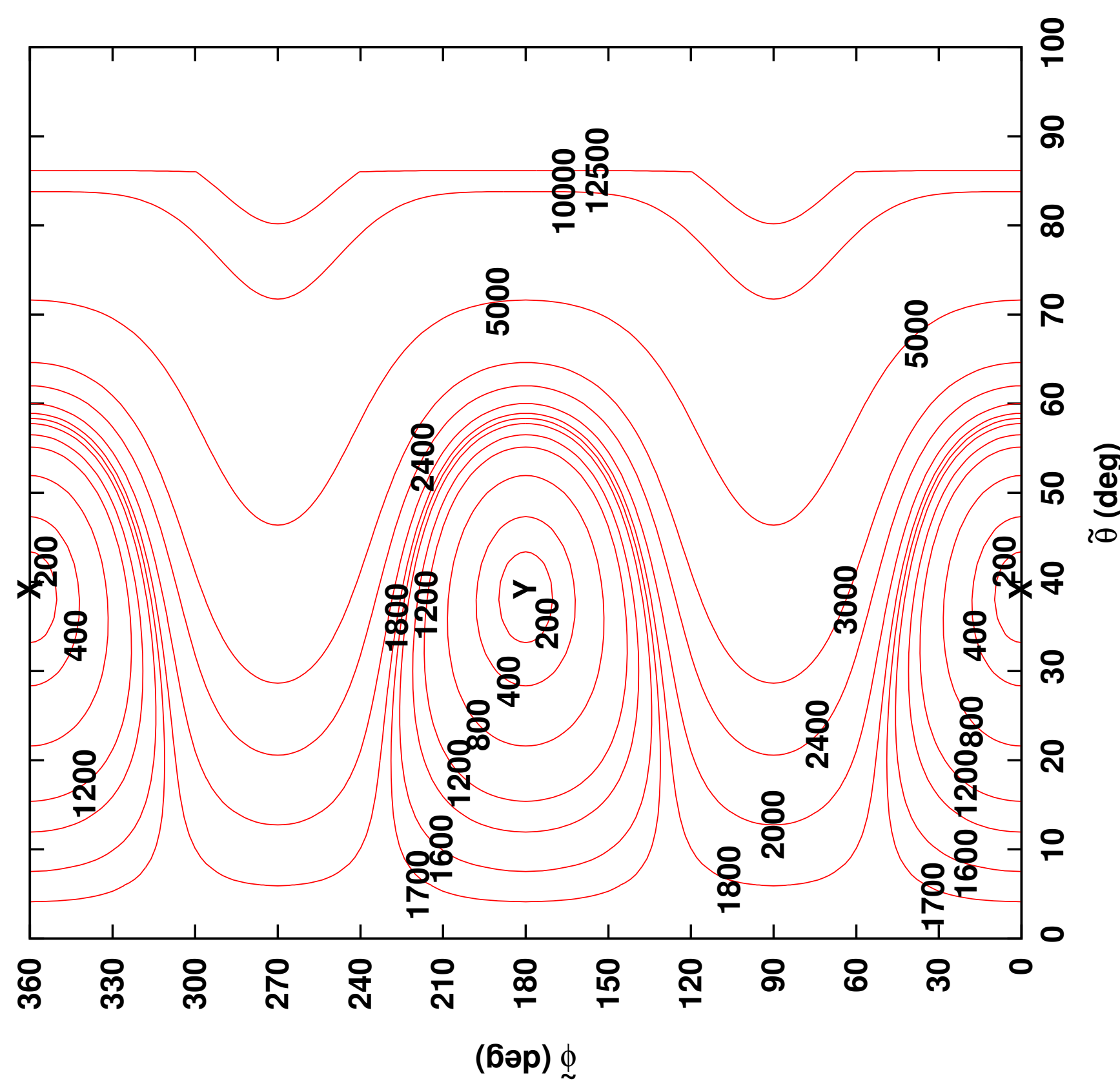




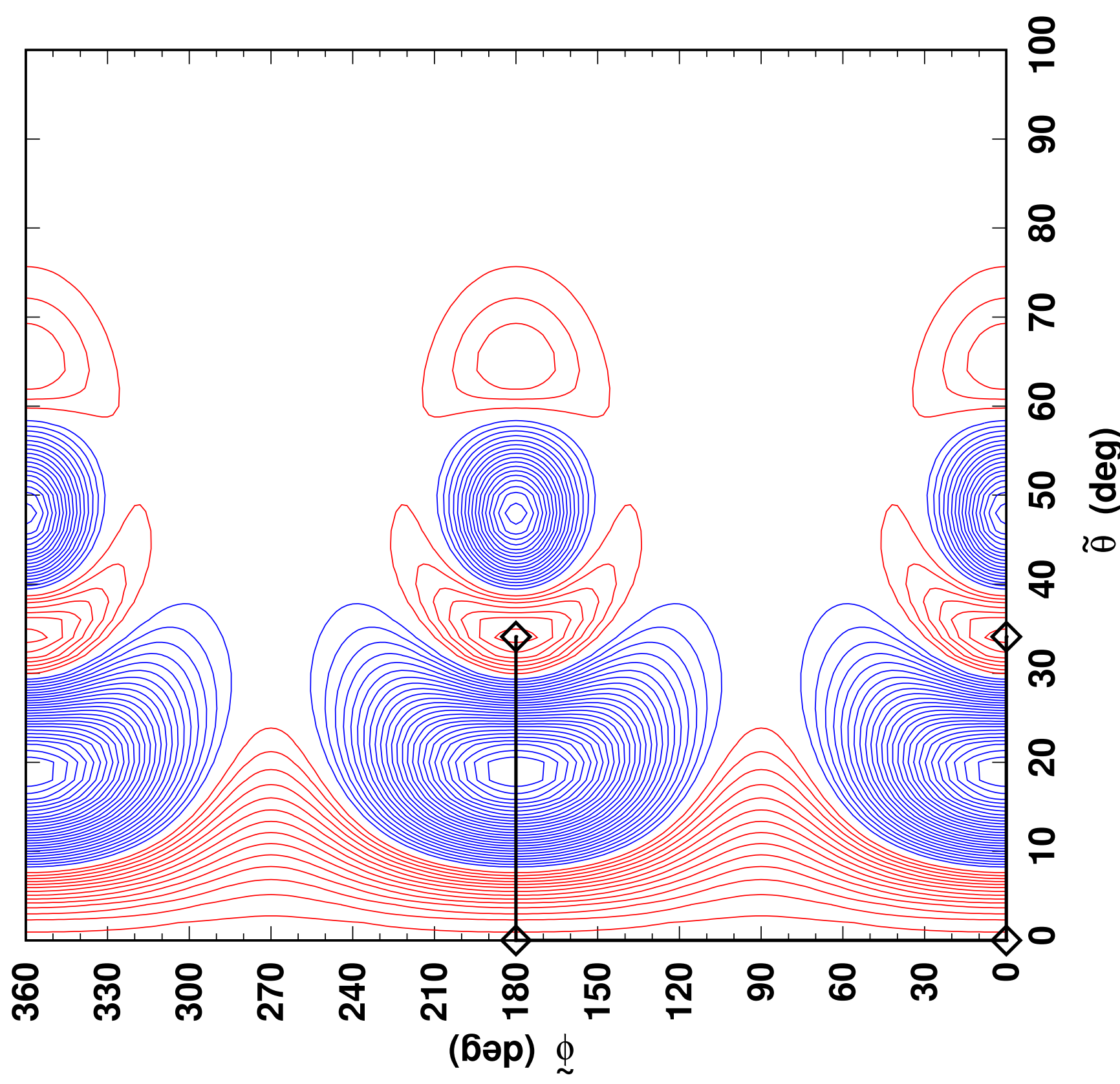




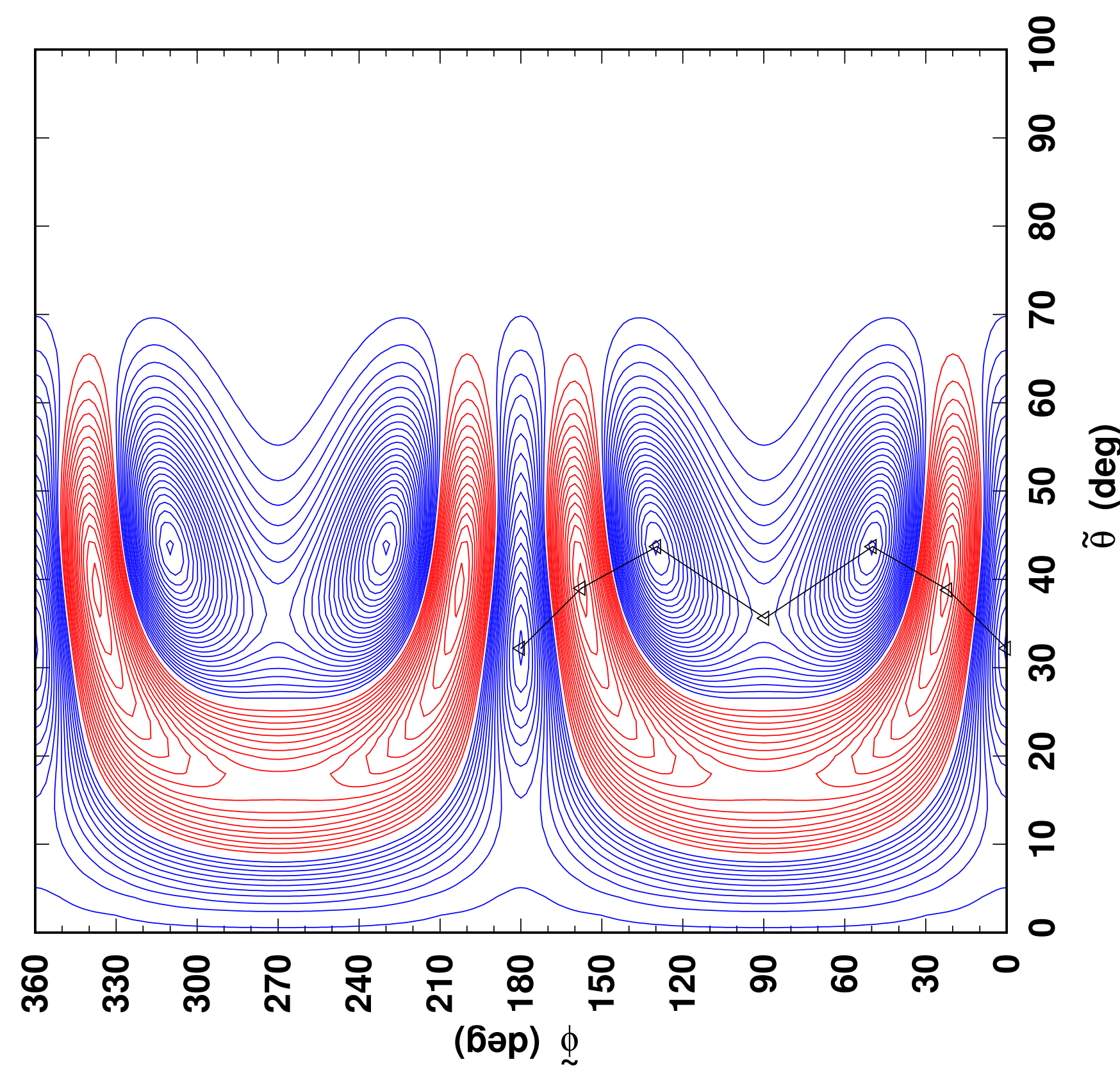




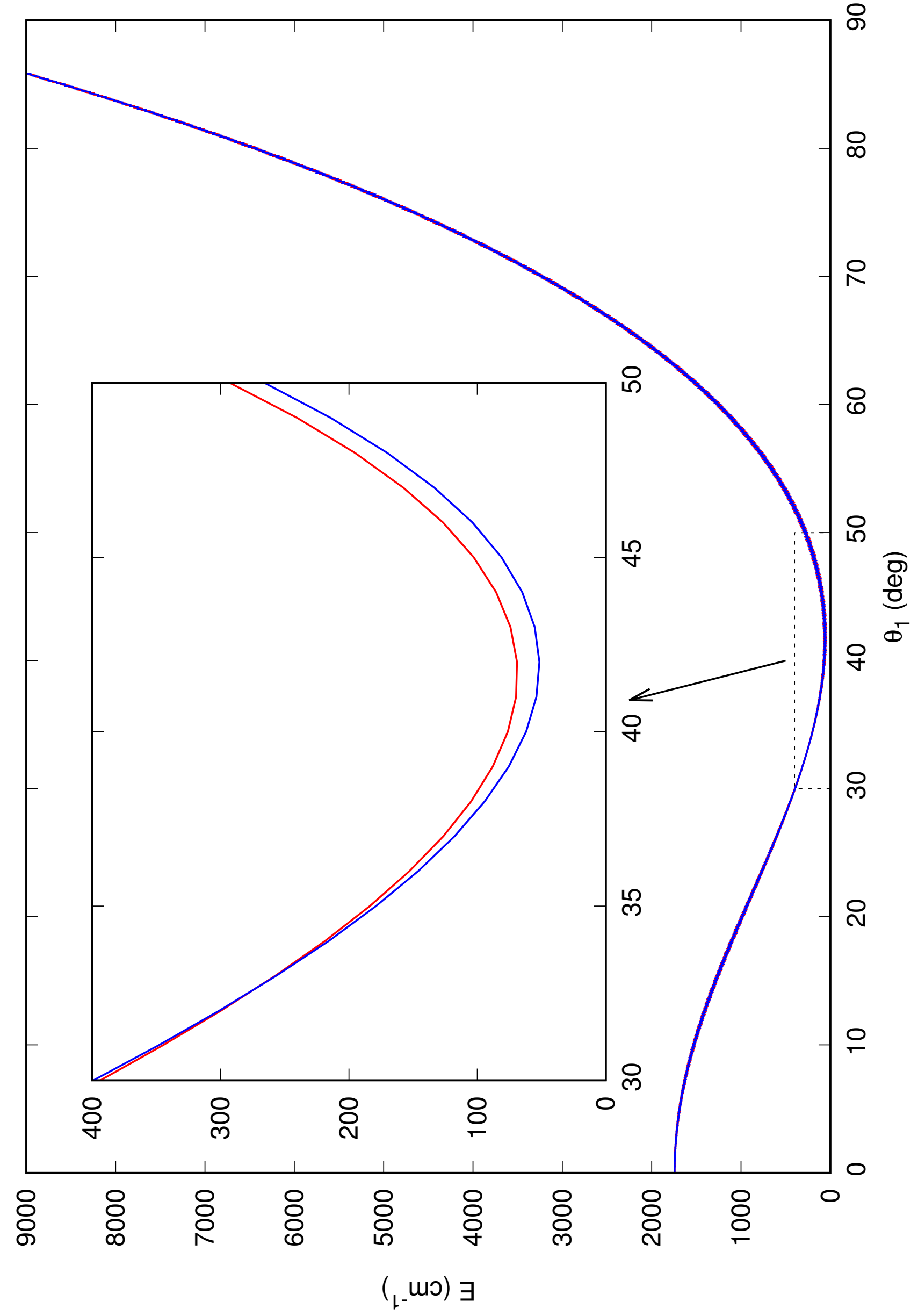

